\title{
3D numerical modelling of twisting cracks under bending and torsion of skew notched beams
}

\author{
Lorenzo Benedetti, Miguel Cervera, Michele Chiumenti \\ International Center for Numerical Methods in Engineering (CIMNE), \\ Technical University of Catalonia (UPC), Edificio C1, Campus Norte, \\ Jordi Girona 1-3, 08034 Barcelona, Spain \\ Corresponding author: lbenedetti@cimne.upc.edu
}

\begin{abstract}
The testing of mode III and mixed mode failure is every so often encountered in the dedicated literature of mechanical characterization of brittle and quasi-brittle materials. In this work, the application of the mixed strain displacement $\boldsymbol{\varepsilon}-\boldsymbol{u}$ finite element formulation to three examples involving skew notched beams is presented. The use of this FE technology is effective in problems involving localization of strains in softening materials.

The objectives of the paper are: (i) to test the mixed formulation in mode III and mixed mode failure and (ii) to present an enhancement in terms of computational time given by the kinematic compatibility between irreducible displacement-based and the mixed strain-displacement elements.

Three tests of skew-notched beams are presented: firstly, a three point bending test of a PolyMethyl MethaAcrylate beam; secondly, a torsion test of a plain concrete prismatic beam with square base; finally, a torsion test of a cylindrical beam made of plain concrete as well. To describe the mechanical behavior of the material in the inelastic range, Rankine and Drucker-Prager failure criteria are used in both plasticity and isotropic continuum damage formats.

The proposed mixed formulation is capable of yielding results close to the experimental ones in terms of fracture surface, peak load and global loss of carrying capability. In addition, the symmetric secant formulation and the compatibility condition between the standard irreducible method and the strain-displacement one is exploited, resulting in a significant speedup of the computational procedure.
\end{abstract}




\section{Introduction}

The experimental testing of brittle and quasi-brittle materials is an exacting and challenging exercise. Three are the failure modes that can be activated: tensile opening, in-plane shearing and out-ofplane shearing. While experimental tests that involve only mode I or mode II are comparatively straightforward to devise, the isolation of mode III represents a challenge. Indeed, this failure type requires the application of a torsion-like load on the specimen but, in reality, it is often impossible to separate mode III from the other two. There is a vast literature that deals specifically with the details of mixed mode tests $[1,2,3,4,5]$ and their analytical solutions $[6,7,8,9,10]$. Quasi brittle materials that fail under tension have the tendency to return to mode I fracture when loaded with a mixed mode stress state. Frequently, this transition takes place because of the curvature of the failure surface and, for this reason, interest is drawn by the shape of the crack propagation.

From the theoretical stand-point, the strength of brittle materials can be predicted by means of Linear Elastic Fracture Mechanics (LEFM), which provides useful quantitative assessment of stress intensity factors and strain energy dissipation near the tip of an evolving crack due to an external load. Nevertheless, LEFM alone is quite limited when addressing elaborated geometries or the progression of the crack tip position. Moreover, it does not provide directly either the shape of the fracture or the global force-displacement behavior. Therefore, the prediction of twisting fracture surfaces in 3D specimens can be only tackled with numerical methods such as the Finite Element Method.

Softening materials exhibiting localization of strains and fracture under external loading are still a strenuous topic in Computational Mechanics. The creation of failure surfaces in a solid body represents, from a mathematical point of view, the inception of a discontinuity in a previously continuous displacement field. Within the FE technology, smearing the localization of strains across a finite length (usually a single element) is an attractive way of avoiding the explicit introduction of discontinuities in the numerical scheme. Once the localization band is smeared across the elements, the dissipation energy becomes dependent on the mesh size and proper energy regularization is necessary for local models to be objective $[11,12]$. For that reason, a considerable effort has been focused on reintroducing the missing length scale in the problem. Non-local $[13,14]$, gradient-enhanced $[15,16]$ micropolar continua $[17,18]$ and phase-field $[19,20]$ are some of the approaches that were proposed in the last three decades. Classically, the problem of mesh size objectivity is overcome by considering explicitly the resolution of the spatial discretization $[12,21]$. However, such straight-forward methodology is held back because standard displacement-based finite elements suffer sensibly from mesh bias and stress locking. The first issue causes the solution to be strongly dependent on the orientation of the computational mesh, with the local lack of convergence affecting the results. The second one is linked to the poor kinematics of standard finite elements, similar to the pressure locking in quasi-incompressible situations. These facts are linked with the limitations of the irreducible formulation and, in turn, they crucially affect energy dissipation and global softening behavior. It is clear that the basic FE technology is not able to deal with propagation of 3D twisting cracks, typical of complex mixed load states.

To take into account the limited capability of the irreducible formulation, several alternative technologies were suggested. Initially, local remeshing of the elements in the vicinity of the crack was used $[22,23]$. Simo $[24,25]$ proposed the enhanced strain elements, which take into account a local decomposition of the strains in compatible and incompatible modes. More recently, the XFEM [26, 27] was introduced as an enrichment of finite elements through the notion of partition of unity. Finally, the strong discontinuity approach $[28,29]$ provides an element formulation that embed the displacement jump in its interior. The implementation of such enrichments requires the use of auxiliary tracking techniques in $2 \mathrm{D}$ and $3 \mathrm{D}$.

Recently, the authors have shown that global and local lack of convergence of the standard displacement-based finite element is the reason for FE spurious mesh biased results. Initially, Cervera et al. [30, 31] proved that avoiding global pressure locking in J2 softening material (both with plasticity and isotropic damage constitutive laws) with the introduction of a proper mixed displacement/pressure $\boldsymbol{u}-p$ formulation leads to mesh-bias independent results for quasi-incompressible localization problems. Then, Cervera et al. $[32,33]$ generalized such concept with the introduction of the strain/displacement 
$\boldsymbol{\varepsilon}-\boldsymbol{u}$ formulation. These formulations were capable to cope with strain softening problems involving isotropic damage [34], quasi-brittle tensile cracking [35], J2 [36] and pressure-dependent plasticity [37] without the need of auxiliary techniques.

The mixed $\boldsymbol{\varepsilon}-\boldsymbol{u}$ finite element formulation is very effective for the solution of linear and nonlinear problems, but it comes at some expense. The simultaneous solution of the displacement and the strain unknown requires larger computational resources. However, it is possible to take advantage of the mathematical structure of the proposed formulation to make important savings on this extra cost.

Therefore, the objective of this work is two-fold. On the one hand, to benchmark the mixed straindisplacement $\boldsymbol{\varepsilon}-\boldsymbol{u}$ formulation in problems involving strain localization and crack propagation under mixed mode I, II, III loading. On the other hand, to exploit the kinematic compatibility between the mixed and irreducible FE formulations to reduce the computational time.

The paper initially presents a summary of the mixed (stabilized) strain/displacement finite elements formulation. The implementation of the method is addressed. Firstly, using a secant formulation yields a symmetric algebraic system to be solved. Secondly, the kinematic compatibility between the irreducible and mixed FE enables the use of the two different formulations on the same mesh in order to save on computational resources. The constitutive laws of isotropic continuum damage and associative plasticity are recalled; both Rankine and Drucker-Prager failure criteria are discussed.

Then, three numerical examples are presented. The first example considers the three point bending test of a PolyMethyl MethAcrylate (PMMA) beam with a skewed 45 degree notch located at the midsection. This first analysis is performed with both irreducible and mixed formulations in order to illustrate the relative benefits of the proposed finite elements technology. The second example tackles the torsion test of a skew-notched prismatic beams with square cross section. The specimen is made of plain concrete and has a centered $45^{\circ}$ notch as well. The objective of this test is to compare the performance of the isotropic continuum damage model or the associative plasticity one using a mode I failure criterion such as Rankine. The third example is a skew-notched cylindrical beam made of plain concrete under torsional load. This test is identical to the previous one except for the geometry of the specimen. Indeed, the different shapes play a major role in the propagation of the localization and the final crack surface. In this case the Drucker-Prager constitutive law is introduced to study the dependence of the experimental results on shear and, consequently, to evaluate mixed mode loading.

Finally, the simultaneous use of irreducible and mixed formulations is benchmarked in terms of computational time and memory requirements. Results show that the proposed solution scheme is capable of saving substantial computational resources while maintaining the same accuracy.

\section{Mixed $\varepsilon-u$ finite elements}

The mixed (stabilized) strain displacement $\boldsymbol{\varepsilon}-\boldsymbol{u}$ finite element method was introduced in Cervera et al. [32] for elasticity and it was extended to isotropic damage constitutive models in Cervera et al. [33] and [34]. The extension to plasticity has been recently presented in Cervera et al. [36] and Benedetti et al. [37], where both incompressible and pressure-dependent plasticity models has been considered for shear-softening materials. In the following, the formulation is briefly recalled in a secant format that can accommodate either continuum damage and plasticity constitutive laws.

The mechanical behavior of a solid body $\mathscr{B}$ occupying the space domain $\Omega$ is described through the compatibility of deformation and the equilibrium of body forces:

$$
\begin{gathered}
-\varepsilon+\nabla^{s} \boldsymbol{u}=\mathbf{0} \\
\nabla \cdot \boldsymbol{\sigma}+\boldsymbol{f}=\mathbf{0}
\end{gathered}
$$

where $\boldsymbol{u}$ is the displacement vector, $\boldsymbol{\varepsilon}$ is the strain tensor, $\boldsymbol{\sigma}$ represents the Cauchy stress tensor, $\boldsymbol{\nabla}^{s}$ and $\boldsymbol{\nabla}$. are the symmetric gradient and the divergence operators respectively, and $\boldsymbol{f}$ is the vector of body forces. The constitutive equation links the strain and stress fields; in the following, a secant form of the system is assumed

$$
\sigma=\mathbb{C}_{s} \varepsilon
$$


where $\mathbb{C}_{s}$ is the secant constitutive tensor. As the strain and stress tensors are symmetric, thermodynamic considerations imply that the secant constitutive tensor needs be symmetric (see Faria et al. [38] for reference). For the isotropic damage model, the constitutive equation reads:

$$
\boldsymbol{\sigma}=(1-d) \mathbb{C}: \varepsilon=\mathbb{C}_{s, d}: \varepsilon
$$

where $d$ is the damage parameter and $\mathbb{C}$ is the elastic constitutive tensor. The damage secant constitutive tensor can be defined as [36]:

$$
\mathbb{C}_{s, d}=(1-d) \mathbb{C}
$$

In case of plasticity, the constitutive equation reads:

$$
\boldsymbol{\sigma}=\mathbb{C}:\left(\varepsilon-\varepsilon_{p}\right)=\mathbb{C}_{s, p}: \varepsilon
$$

where $\varepsilon_{p}$ is the plastic strain tensor. The plastic secant constitutive tensor is defined as:

$$
\mathbb{C}_{s, p}=\mathbb{C}-\frac{\left(\mathbb{C}: \varepsilon_{p}\right) \otimes\left(\mathbb{C}: \varepsilon_{p}\right)}{\varepsilon: \mathbb{C}: \varepsilon_{p}}
$$

Note that both secant constitutive tensors, equations (4) and (6), are symmetric.

The strong form of the boundary value problem is completed by imposing proper boundary conditions on $\partial \Omega$ and providing the evolution laws for the plastic strain tensor $\varepsilon_{p}$ or for the damage variable $d$.

After symmetrizing the system of equations by pre-multiplication of the secant constitutive tensor $\mathbb{C}_{s}$, the strong form of the mixed problem in the unknown fields of total strains $\varepsilon$ and displacements $\boldsymbol{u}$ reads:

$$
\begin{gathered}
-\mathbb{C}_{s}: \varepsilon+\mathbb{C}_{s}: \nabla^{s} \boldsymbol{u}=\mathbf{0} \\
\nabla \cdot\left[\mathbb{C}_{s}: \varepsilon\right]+\boldsymbol{f}=\mathbf{0}
\end{gathered}
$$

Now, equation (7a) represents compatibility of deformation and constitutive behavior while equation (7b) represents equilibrium. The corresponding weak form is obtained by introducing the test functions $\gamma$ for strains and $\boldsymbol{v}$ for displacements, respectively, pertaining to the functional spaces $\mathbb{G} \subset L^{2}(\Omega)^{d i m}$ and $\mathbb{V} \subset H^{1}(\Omega)^{d i m}$. Hence, applying Gauss's divergence theorem to the equilibrium equation, the weak form of the mixed problem is:

$$
\begin{gathered}
-\int_{\Omega} \gamma: \mathbb{C}_{s}: \varepsilon+\int_{\Omega} \gamma: \mathbb{C}_{s}: \nabla^{s} \boldsymbol{u}=0 \quad \forall \boldsymbol{\gamma} \in \mathbb{G} \\
\int_{\Omega} \boldsymbol{\nabla}^{s} \boldsymbol{v}:\left[\mathbb{C}_{s}: \boldsymbol{\varepsilon}\right]=F(\boldsymbol{v}) \quad \forall \boldsymbol{v} \in \mathbb{V}
\end{gathered}
$$

where the term $F(\boldsymbol{v})$ represents the work done by tractions on $\partial \Omega$ and body forces in $\Omega$. Note that this continuous weak form is symmetric.

The discrete FE version of the mixed weak form of the problem is found by substituting the unknown fields with their finite element interpolation counterparts:

$$
\begin{gathered}
\boldsymbol{\varepsilon} \rightarrow \boldsymbol{\varepsilon}_{h}=\sum_{i=1}^{n_{p t s}} \gamma_{h}^{(i)} \varepsilon_{h}^{(i)} \quad \gamma_{h} \in \mathbb{G}_{h} \\
\boldsymbol{u} \rightarrow \boldsymbol{u}_{h}=\sum_{i=1}^{n_{p t s}} \boldsymbol{v}_{h}^{(i)} \boldsymbol{u}_{h}^{(i)} \quad \boldsymbol{v}_{h} \in \mathbb{V}_{h}
\end{gathered}
$$

where $\boldsymbol{\varepsilon}_{h}$ and $\boldsymbol{u}_{h}$ are the nodal degrees of freedom whereas $\gamma_{h}$ and $\boldsymbol{v}_{h}$ are the discrete interpolation functions for the strain and the displacement fields pertaining respectively to the spaces $\mathbb{G}_{h}$ and $\mathbb{V}_{h}$, the discrete counterparts of $\mathbb{G}$ and $\mathbb{V}$. 
The choice of finite elements in the discretization is crucial for the necessary stability of the employed numerical scheme, e.g. [39, 40, 41, 42]. In particular, the Inf-Sup condition proves that equal interpolations for strains and displacements (such as P1P1) are bound to be unstable. A stabilization procedure is then required: a modification of the discrete variational form provides the numerical stability, while maintaining consistency. Using the Variational Multiscale Stabilization procedure $[43,44]$ as presented in $[36,37]$, the set of equations for the stabilized problem reads:

$$
\begin{gathered}
-\left(1-\tau_{\varepsilon}\right) \int_{\Omega} \gamma_{h}: \mathbb{C}_{s}:\left(\boldsymbol{\varepsilon}_{h}-\boldsymbol{\nabla}^{s} \boldsymbol{u}_{h}\right) \\
\quad-\tau_{u} \int_{\Omega}\left[\boldsymbol{\nabla} \cdot\left(\mathbb{C}_{s}: \boldsymbol{\gamma}_{h}\right)\right] \cdot\left[\boldsymbol{\nabla} \cdot\left[\mathbb{C}_{s}: \boldsymbol{\varepsilon}_{h}\right]+f\right]=0 \quad \forall \boldsymbol{\gamma}_{h} \in \mathbb{G}_{h} \\
\int_{\Omega} \boldsymbol{\nabla}^{s} \boldsymbol{v}_{h}: \mathbb{C}_{s}: \varepsilon_{h}-\tau_{\boldsymbol{\varepsilon}} \int_{\Omega} \boldsymbol{\nabla}^{s} \boldsymbol{v}_{h}: \mathbb{C}_{s}:\left[\varepsilon_{h}-\boldsymbol{\nabla}^{s} \boldsymbol{u}_{h}\right]=F\left(\boldsymbol{v}_{h}\right) \quad \forall \boldsymbol{v}_{h} \in \mathbb{V}_{h}
\end{gathered}
$$

The scalars $\tau_{\boldsymbol{\varepsilon}}$ and $\tau_{\boldsymbol{u}}$ are the stabilization parameter computed as:

$$
\tau_{\varepsilon}=c_{\varepsilon} \frac{h}{L_{0}} \quad \tau_{\boldsymbol{u}}=c_{\boldsymbol{u}} \frac{h L_{0}}{E}
$$

where $c_{\boldsymbol{u}}$ and $c_{\boldsymbol{\varepsilon}}$ are arbitrary positive numbers, $E$ is the Young's modulus, $h$ is the representative size of the finite element mesh and $L_{0}$ is a characteristic length of the problem.

The stabilized formulation is consistent with the original discrete weak form since, with converging values of the unknowns $\varepsilon_{h}$ and $\boldsymbol{u}_{h}$, the contribution of the stabilization terms (those multiplied by $\tau_{\varepsilon}$ and $\tau_{\boldsymbol{u}}$ ) disappears, being dependent on the residuals of the strong form of the problem, respectively (see equations $(7 \mathrm{a})$ and $(7 \mathrm{~b})$ ):

$$
r_{\varepsilon_{h}}=\mathbb{C}_{s}: \varepsilon_{h}-\mathbb{C}_{s}: \nabla^{s} \boldsymbol{u}_{h} \quad r_{\boldsymbol{u}_{h}}=\boldsymbol{\nabla} \cdot\left[\mathbb{C}_{s}: \varepsilon_{h}\right]+\boldsymbol{f}
$$

When dealing with problems that do not involve incompressibility constrains, it is possible to drop the displacement subscale and consider solely the strain one by setting $\tau_{\boldsymbol{u}}=0$. The final set of equations for the mechanical problem reads:

$$
\begin{gathered}
-\left(1-\tau_{\boldsymbol{\varepsilon}}\right) \int_{\Omega} \gamma_{h}: \mathbb{C}_{s}:\left(\varepsilon_{h}-\nabla^{s} \boldsymbol{u}_{h}\right)=0 \quad \forall \gamma_{h} \in \mathbb{G}_{h} \\
\int_{\Omega} \boldsymbol{\nabla}^{s} \boldsymbol{v}_{h}: \mathbb{C}_{s}: \varepsilon_{h}-\tau_{\boldsymbol{\varepsilon}} \int_{\Omega} \boldsymbol{\nabla}^{s} \boldsymbol{v}_{h}: \mathbb{C}_{s}:\left[\boldsymbol{\varepsilon}_{h}-\nabla^{s} \boldsymbol{u}_{h}\right]=F\left(\boldsymbol{v}_{h}\right) \quad \forall \boldsymbol{v}_{h} \in \mathbb{V}_{h}
\end{gathered}
$$

Furthermore, expression (13b) can be written as:

$$
\int_{\Omega} \boldsymbol{\nabla}^{s} \boldsymbol{v}_{h}: \mathbb{C}_{s}: \varepsilon_{s t a b, h}=F\left(\boldsymbol{v}_{h}\right) \quad \forall \boldsymbol{v}_{h} \in \mathbb{V}_{h}
$$

where the stabilized discrete strain field

$$
\varepsilon_{s t a b, h}=\left(1-\tau_{\varepsilon}\right) \varepsilon_{h}+\tau_{\varepsilon} \nabla^{s} \boldsymbol{u}_{h}
$$

is a blending of the continuous $\left(\varepsilon_{h}\right)$ and discontinuous $\left(\boldsymbol{\nabla}^{s} \boldsymbol{u}_{h}\right)$ strain fields weighted by the stabilization parameter $\tau_{\varepsilon}$. The discrete stabilized form, equation (13), is symmetric.

\section{Compatibility with standard u finite elements}

The mechanical problem is governed by the compatibility equation (1a), the equilibrium equation (1b) and the constitutive equation (2), all in strong form. As described above, the variational mixed $\boldsymbol{\varepsilon}-\boldsymbol{u}$ form, in equations (8a)-(8b), takes $\boldsymbol{\varepsilon}$ and $\boldsymbol{u}$ as main variables and considers both compatibility and 

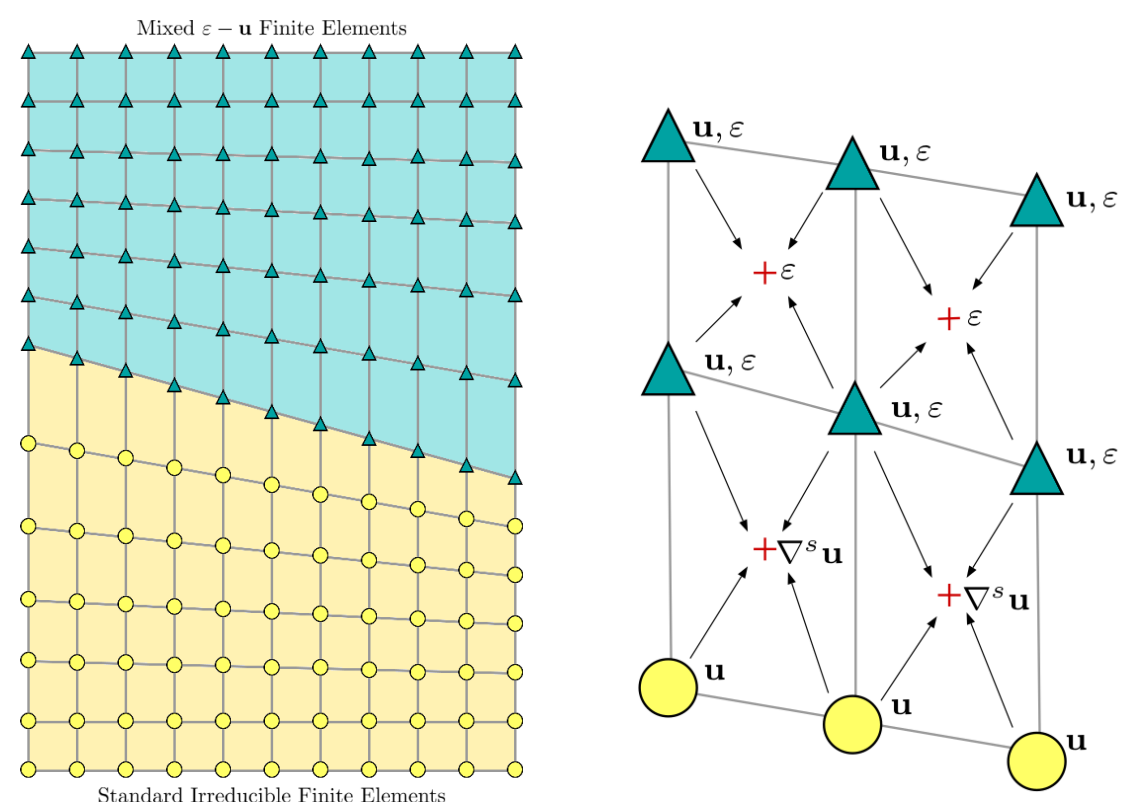

Figure 1: FE mesh with combined standard and mixed formulations. Turquoise color represents the $\boldsymbol{\varepsilon}-\boldsymbol{u}$ elements whereas yellow represents the displacement-based ones. The strain tensor at the Gauss points (symbolized with red crosses) is computed with the interpolation of nodal strain in the mixed formulation or the discrete symmetric gradient of displacements in the irreducible one.

equilibrium in weak form. The corresponding discrete $\mathrm{FE}$ form requires the interpolation of both the strain and the displacement fields, with $\varepsilon_{h}$ and $\boldsymbol{u}_{h}$ as nodal degrees of freedom.

Alternatively, the more standard irreducible $\boldsymbol{u}$ form takes only the displacement as main variable and considers only equilibrium in weak form. To this end, substituting equation (1a) into equation $(2)$, and this into equation (1b), yields:

$$
\boldsymbol{\nabla} \cdot\left[\mathbb{C}_{s}: \nabla^{s} \boldsymbol{u}\right]+\boldsymbol{f}=0
$$

with the corresponding variational (weak) form

$$
\int_{\Omega} \boldsymbol{\nabla}^{s} \boldsymbol{v}: \mathbb{C}_{s}: \boldsymbol{\nabla}^{s} \boldsymbol{u}=F(\boldsymbol{v}) \quad \forall \boldsymbol{v} \in \mathbb{V}
$$

The irreducible discrete FE form requires solely the interpolation of the displacement field:

$$
\boldsymbol{u} \rightarrow \boldsymbol{u}_{h}=\sum_{i=1}^{n_{p t s}} \boldsymbol{v}_{h}^{(i)} \boldsymbol{u}_{h}^{(i)} \quad \boldsymbol{v}_{h} \in \mathbb{V}_{h}
$$

with $\boldsymbol{u}_{h}$ as the nodal degrees of freedom, which is identical to equation (9b).

From a computational perspective, the $\boldsymbol{\varepsilon}-\boldsymbol{u}$ finite element presents a larger number of variables to be solved compared to the standard one. For each mesh node of a 3D problem, the vector of unknowns contains 9 scalars, 3 displacements $\left(u_{x}, u_{y}, u_{z}\right)$ and 6 strains $\left(\varepsilon_{x x}, \varepsilon_{y y}, \varepsilon_{z z}, \varepsilon_{x y}, \varepsilon_{x z}, \varepsilon_{y z}\right)$ of the symmetric deformation tensor, in Voigt's notation.

Note that, if the same interpolation functions $\boldsymbol{u}_{h}$ are selected, the kinematics of the mixed and the irreducible formulations are compatible, i.e. the requirement of inter-elemental continuity is satisfied. This is necessary to prove consistency of the FE form in the classical Rayleigh-Ritz sense. Therefore, a mesh constructed as in Figure 1, where the top part is formed by mixed $\boldsymbol{\varepsilon}-\boldsymbol{u}$ elements while the bottom part is made of standard $\boldsymbol{u}$ ones, is feasible.

Indeed, the standard finite elements are a particular case of the stable mixed formulation, see equation (14). Setting $\tau_{\varepsilon}=1$ in expressions (13), equation (13a) becomes an identity and (13b) reduces to (17). 
Therefore, it is possible to reduce the computational burden by considering a combined standard/mixed FE mesh. Setting the stabilization parameter $\tau_{\varepsilon}=1$ where possible and skipping the corresponding elemental computations leads to substantial savings in the total number of degrees of freedom, global operations and corresponding matrix storage.

\section{Algebraic implementation aspects}

In previous works $[35,36,37]$, the nonlinear algebraic problem in equations (13a)-(13b) was solved in an incremental-iterative manner using the Newton-Raphson method.

Let the algebraic nonlinear problem be written in an incremental-iterative fashion as

$$
\boldsymbol{R}\left(\boldsymbol{X}_{n+1}^{i+1}\right)=\boldsymbol{P}\left(\boldsymbol{X}_{n+1}^{i+1}\right)-\boldsymbol{F}_{n+1}=\mathbf{0}
$$

where $n$ and $i$ are the increment and iteration counters, respectively; $\boldsymbol{X}$ is the solution vector, $\boldsymbol{P}, \boldsymbol{F}$ and $\boldsymbol{R}$ are the internal, external and residual force vectors.

Writing an iterative correction as:

$$
\boldsymbol{X}_{n+1}^{i+1}=\boldsymbol{X}_{n+1}^{i}+\delta \boldsymbol{X}^{i+1}
$$

and given that a linear Taylor's approximation of the internal forces

$$
\boldsymbol{R}\left(\boldsymbol{X}_{n+1}^{i+1}\right) \simeq \boldsymbol{R}\left(\boldsymbol{X}_{n+1}^{i}\right)+\boldsymbol{J}\left(\boldsymbol{X}_{n+1}^{i}\right) \delta \boldsymbol{X}^{i+1}=\mathbf{0}
$$

where $\boldsymbol{J}\left(\boldsymbol{X}_{n+1}^{i}\right)$ is the jacobian (tangent) matrix. It follows that

$$
\boldsymbol{J}\left(\boldsymbol{X}_{n+1}^{i}\right) \delta \boldsymbol{X}^{i+1}=-\boldsymbol{R}\left(\boldsymbol{X}_{n+1}^{i}\right)
$$

This is Newton-Raphson's method. On the one hand, this procedure presents asymptotic quadratic convergence when exact tangent matrices are used and the initial approximation is close enough to the solution; on the other hand, this results in a non-symmetric algebraic system to be solved. The reason for the non-symmetry of the rate problem derived from equations (13) are (i) the (possible) lack of symmetry of the constitutive tangent tensor and (ii) the non-symmetric dependence of the discrete stresses on $\varepsilon_{h}$ and $\boldsymbol{u}_{h}$.

Alternatively, the nonlinear algebraic problem in equations (13) may be solved using the secant (or Picard's) method.

Let the internal forces be written in secant form as

$$
\boldsymbol{P}\left(\boldsymbol{X}_{n+1}^{i+1}\right)=\boldsymbol{S}\left(\boldsymbol{X}_{n+1}^{i+1}\right) \boldsymbol{X}_{n+1}^{i+1}
$$

Given that

$$
\boldsymbol{R}\left(\boldsymbol{X}_{n+1}^{i}\right)=\boldsymbol{S}\left(\boldsymbol{X}_{n+1}^{i}\right) \boldsymbol{X}_{n+1}^{i}-\boldsymbol{F}_{n+1}
$$

it follows that

$$
\boldsymbol{S}\left(\boldsymbol{X}_{n+1}^{i}\right) \delta \boldsymbol{X}^{i+1}=-\boldsymbol{R}\left(\boldsymbol{X}_{n+1}^{i}\right)
$$

The secant method converges superlinearly, but the need of evaluating consistent derivatives is avoided. Furthermore, in the case under consideration, the secant matrix $\boldsymbol{S}\left(\boldsymbol{X}_{n+1}^{i}\right)$ is symmetric.

Comparing expressions (22) and (25) it is obvious that both methods can be implemented similarly, the only difference being the use of the tangent or secant matrix.

A further approximation can be used in both methods by making $\boldsymbol{J}\left(\boldsymbol{X}_{n+1}^{i}\right) \simeq \boldsymbol{J}\left(\boldsymbol{X}_{n+1}^{1}\right)$ and $\boldsymbol{S}\left(\boldsymbol{X}_{n+1}^{i}\right) \simeq \boldsymbol{S}\left(\boldsymbol{X}_{n+1}^{1}\right)$ with the matrices recomputed only for the first iteration of the increments. These are modified Newton-Raphson's and Picard's schemes. The convergence is linear for both methods. 
For the mixed finite element formulation discussed in Section $2, \boldsymbol{X}_{n+1}=\left[\boldsymbol{\varepsilon}_{h}, \boldsymbol{u}_{h}\right]_{n+1}^{T}$. Details on the algebraic tangent system of equations (22) are given in references [37,36]. The algebraic secant system of equations (25) reads:

$$
\left[\begin{array}{cc}
\boldsymbol{M}_{\tau} & \boldsymbol{G}_{\tau} \\
\boldsymbol{D}_{\tau} & \boldsymbol{K}_{\tau}
\end{array}\right]_{n}^{i}\left[\begin{array}{l}
\delta \boldsymbol{\varepsilon}_{h} \\
\delta \boldsymbol{u}_{h}
\end{array}\right]_{n+1}^{i+1}=-\left[\begin{array}{c}
R_{1, h} \\
R_{2, h}
\end{array}\right]_{n+1}^{i}
$$

and the submatrices $\boldsymbol{M}_{\tau}, \boldsymbol{G}_{\tau}, \boldsymbol{D}_{\tau}$ and $\boldsymbol{K}_{\tau}$ are computed as:

$$
\begin{gathered}
\boldsymbol{M}_{\tau}=-\left(1-\tau_{\boldsymbol{\varepsilon}}\right) \int_{\Omega} \boldsymbol{N}_{\boldsymbol{\varepsilon}}^{T} \mathbb{C}_{s} \boldsymbol{N}_{\boldsymbol{\varepsilon}}-\tau_{\boldsymbol{u}} \int_{\Omega} \mathbb{C}_{s} \boldsymbol{B} \boldsymbol{B}^{T} \mathbb{C}_{s} \\
\boldsymbol{G}_{\tau}=\left(1-\tau_{\boldsymbol{\varepsilon}}\right) \int_{\Omega} \boldsymbol{N}_{\boldsymbol{\varepsilon}}^{T} \mathbb{C}_{s} \boldsymbol{B} \\
\boldsymbol{D}_{\tau}=\left(1-\tau_{\boldsymbol{\varepsilon}}\right) \int_{\Omega} \boldsymbol{B}^{T} \mathbb{C}_{s} \boldsymbol{N}_{\boldsymbol{u}} \\
\boldsymbol{K}_{\tau}=\tau_{\boldsymbol{\varepsilon}} \int_{\Omega} \boldsymbol{B}^{T} \mathbb{C}_{s} \boldsymbol{B}
\end{gathered}
$$

where $\boldsymbol{M}$ is the mass-like projection matrix, $\boldsymbol{G}$ is the discrete gradient matrix, $\boldsymbol{D}$ is the discrete divergence matrix and $\boldsymbol{K}$ is the stiffness matrix.

\section{$5 \quad$ Plasticity and damage models}

In this work, both plasticity and damage models are introduced to describe strain localization and failure. Both Rankine and Drucker-Prager failure criteria are used. Figure 2 shows a representation of the corresponding admissible elastic domains in the Haigh-Westergaard space.

In this space of principal stresses, the Rankine criterion appears as the intersection of three mutually orthogonal planes, which are, at the same time, orthogonal to the principal axes. Therefore, it is a triangular pyramid with the apex located on the hydrostatic axis. Contrariwise, the Drucker-Prager criterion appears as a cone with a circular cross-section in the octahedral plane and axisymmetric to the $\sigma^{1}=\sigma^{2}=\sigma^{3}$ axis. Both criteria are pressure-dependent, although the Drucker-Prager criterion reduces to the pressure-independent Von Mises one for vanishing friction angle.

In Table 1, a general overview of the implemented constitutive laws is presented. The failure criterion is defined by the scalar function $f(\boldsymbol{\sigma}, q)$ which depends on the equivalent stress measure
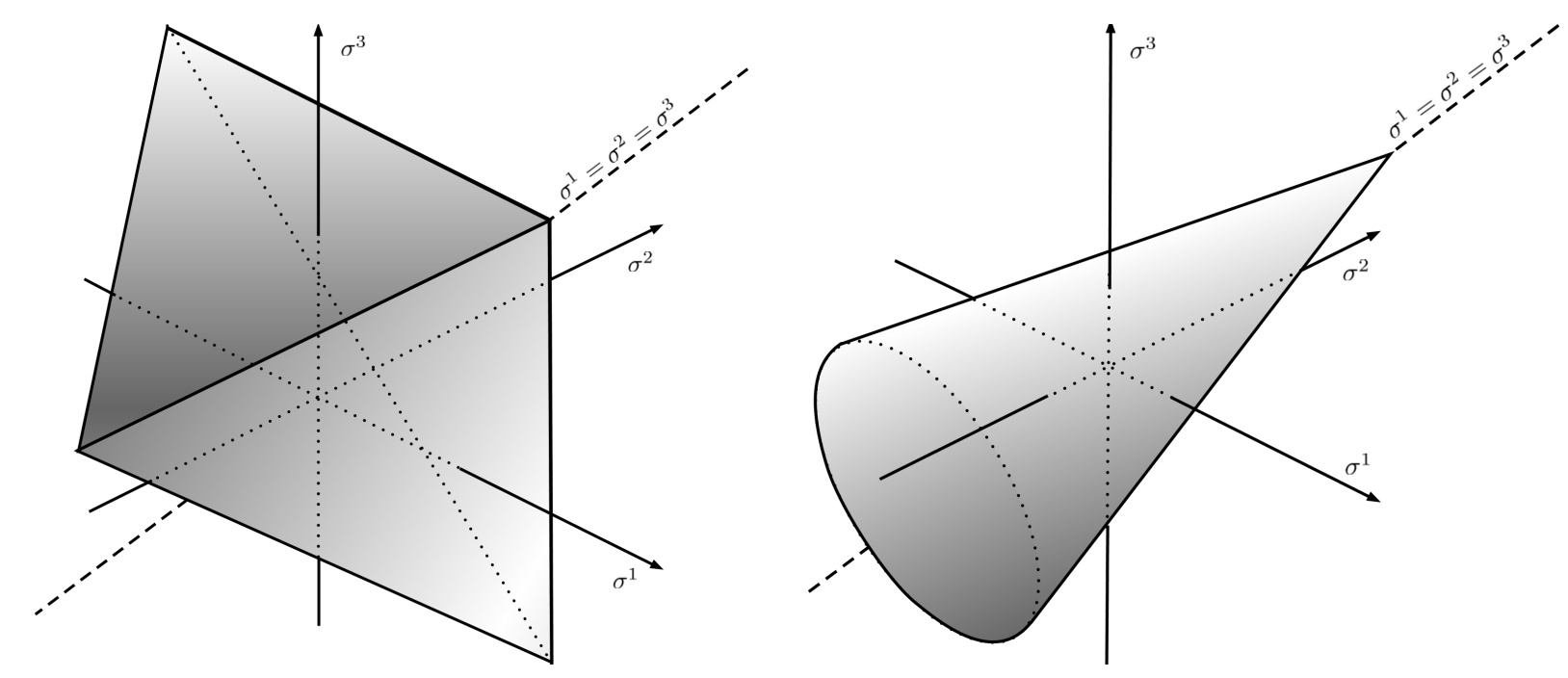

Figure 2: Representation of the Rankine and Drucker-Prager failure criteria in the principal stress space $\left(\sigma^{1}, \sigma^{2}, \sigma^{3}\right)$. 
$\tau(\boldsymbol{\sigma})$ and the stress-like isotropic softening function $q(\xi) ; \xi$ is the strain-like softening variable which controls the evolution of the failure surface.

In the case of the Rankine failure criterion, the equivalent stress is given by the first principal stress value as:

$$
\tau(\boldsymbol{\sigma})=\sigma_{1}
$$

whereas, in the Drucker-Prager failure criterion, shear stress and pressure are linearly combined through the tangent of the friction angle $\phi$ :

$$
\tau(\boldsymbol{\sigma})=\sqrt{3 J_{2}(\boldsymbol{\sigma})}+\frac{1}{3} I_{1}(\boldsymbol{\sigma}) \tan (\phi)
$$

being $I_{1}(\boldsymbol{\sigma})$ the trace of the stress tensor and $J_{2}(\boldsymbol{\sigma})$ the second invariant of the deviatoric part of $\boldsymbol{\sigma}$.

Despite having identically failure criteria and being their evolution controlled by analogous loadingunloading conditions (Karush-Kuhn-Tucker conditions), the global behavior of the two constitutive models is substantially different. Inelastic flow in plasticity is directional; in particular, in the associative case, it is defined by the normal vector to the yield surface. Therefore, for Rankine-type plastic models, the inelastic flow occurs strictly parallel to one of the principal axes. This does not occur for the Drucker-Prager model, where plastic flow is orthogonal to the cone in Figure 2. In marked contrast, inelastic deformation in the continuum isotropic damage model is not directional, since it affects equally all directions of the Haigh-Westergaard space. As shown later, this has a large influence on strain localization and failure when softening behavior is considered.

In the following, exponential softening is considered for both the plastic and continuum damage models. Note that, in a softening process, the energy dissipated by the inelastic behavior is linked to the fracture energy $G_{f}$, defined by unit surface. However, when using a plastic or damage model, the dissipated energy is defined by unit volume. These two definitions are related through a characteristic length $l_{c h}$ in the discrete FE setting, which, in turn, depends on the resolution of the mesh. The size of the strain concentration band depends on the finite element technology, as pointed out by Cervera et al. [33]: irreducible finite elements provide a concentration band within a single element span, having a discontinuos strain field; contrariwise, in the $\boldsymbol{\varepsilon}-\boldsymbol{u}$ mixed FE formulation, the slip line spans two elements due to inter-elemental continuous strain. Consequently, the characteristic length $l_{c h}$ is taken accordingly. Additional details of the models can be found in references [37] for the Drucker-Prager plasticity model, [35] for the Rankine plasticity model and [34, 45, 46] for the Rankine isotropic damage model.

Associative plasticity model Isotropic continuum damage model

$\begin{array}{ll}\text { Constitutive equation } \quad \boldsymbol{\sigma}=\mathbb{C}:\left(\varepsilon-\varepsilon_{p}\right) & \boldsymbol{\sigma}=(1-d) \mathbb{C}: \boldsymbol{\varepsilon}\end{array}$

Softening function

$$
q=q(\xi)
$$

Inelastic criterion

$$
f(\boldsymbol{\sigma}, q)=\tau(\boldsymbol{\sigma})-\left(\sigma_{y}-q\right)
$$

Internal variables evolution

$$
\dot{\xi}=\dot{\lambda}, \quad \dot{\varepsilon}_{p}=\dot{\lambda} \frac{\partial f}{\partial \sigma}
$$$$
\dot{\xi}=\dot{\lambda}, \quad d(\xi)=1-\frac{q}{\xi}
$$

Loading-unloading conditions

$$
\dot{\lambda} \geq 0, \quad f(\boldsymbol{\sigma}, q) \leq 0, \quad \dot{\lambda} f(\boldsymbol{\sigma}, q)=0
$$

Table 1: Summary of associative plasticity and isotropic continuum damage models. Both Rankine and Drucker-Prager failure criteria are considered. 


\section{$6 \quad$ Numerical Simulations}

\subsection{Three point bending test on skew notched beam}

The first example is a three point bending test on a beam with a slanted notch. The slot is vertical, with an inclination of $45^{\circ}$ with respect to the longitudinal midplane of the beam. The specimen geometry, represented in Figure 3, has a total length $L=260 \mathrm{~mm}$, effectively supported span $L_{e}=240 \mathrm{~mm}$, by a thickness $t=10 \mathrm{~mm}$ and a total height $w=60 \mathrm{~mm}$. The initial notch is $a=20 \mathrm{~mm}$ high $(a=w / 3)$, with a constant section width of $2 \mathrm{~mm}$ and a slant angle $\gamma=45^{\circ}$.

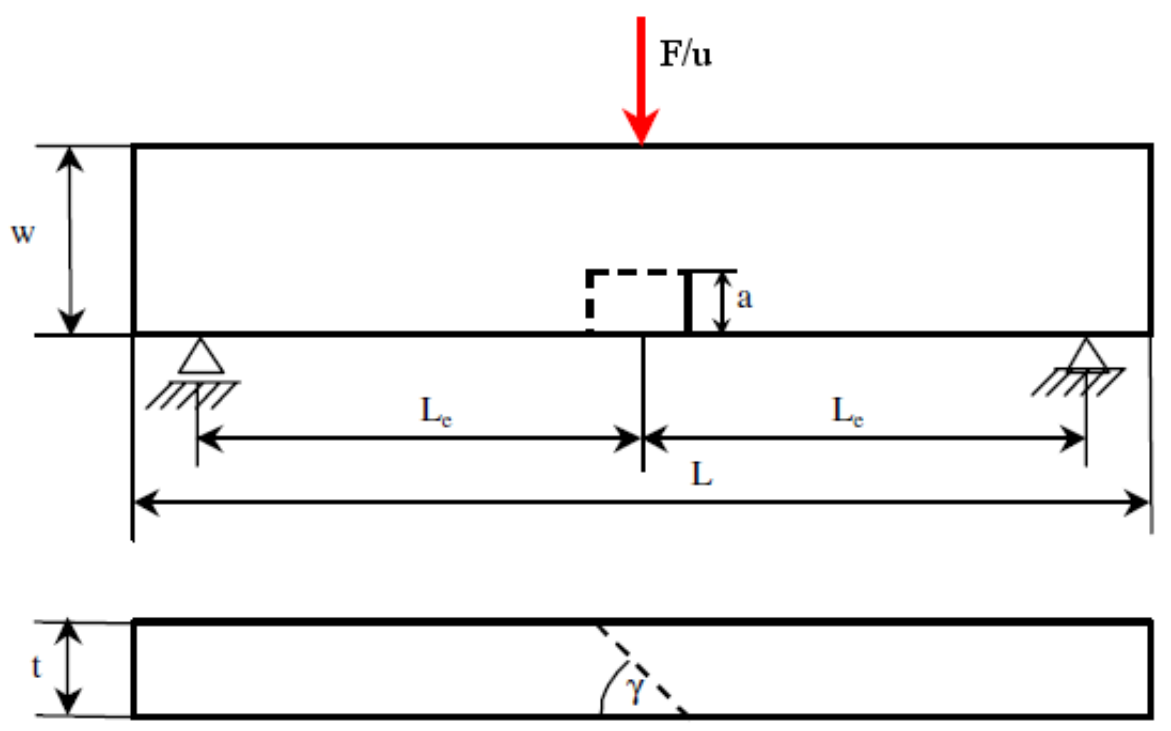

Figure 3: Geometry of the twisted crack 3PB test, taken from Citarella and Buchholz [47]

This test was initially introduced by Pook [1] to study the propagation of the crack front under cyclic loading in steel specimens. More recently, the same tests were recreated by Cooke and Pollard [5], Buchholz et al. [9] using PolyMethyl MethAcrylate (PMMA), also known as Plexiglass, in order to better examine the crack front evolution through its transparency. Lazarus and Leblond [6] and Lazarus et al. [8] studied the same problem in the case of monotonic load. Finally, Citarella and Buchholz [47] and Ferté et al. [48] studied the problem from a computational stand point using the Boundary Elements Method and the X-FEM technology, respectively.

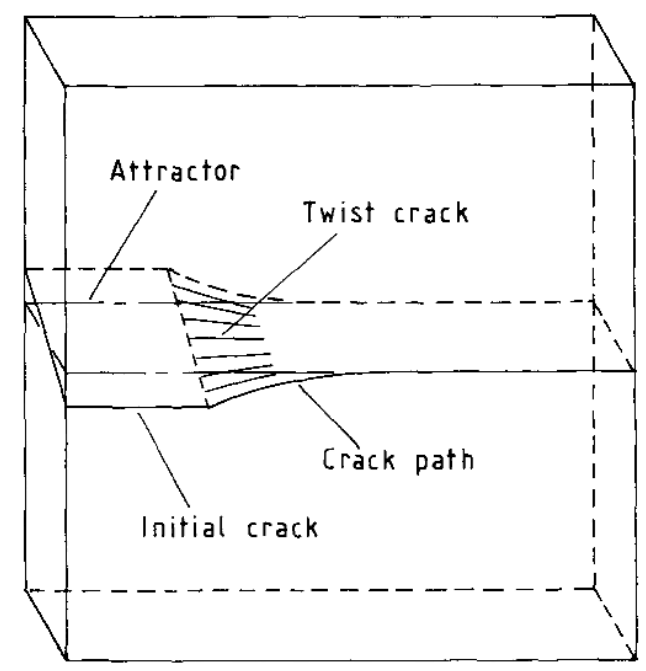

Figure 4: Crack path interpretation from Pook [3] 


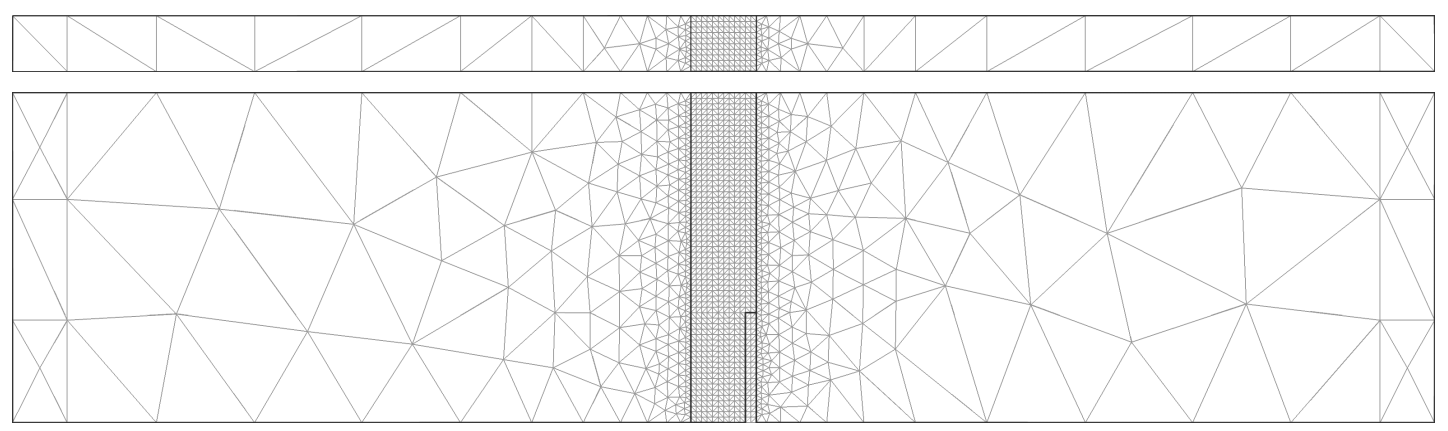

Figure 5: Computational mesh of $\mathrm{P} 1$ and $\mathrm{P} 1 \mathrm{P} 1$ tetrahedral elements used for the 3 point bending test.

Examining the experimental results (Figure 4), the crack starts from the initial notch and, with increasing applied load, a rotation of the failure surface is observable. Pook [3] and Yates and Mohammed [4] showed that this characteristic behavior is due to the transition from Mode III to Mode I fracture. At the beginning of the test, the stresses in the vicinity of the notch are given by the asymmetrical bending of the specimen, resulting in a diagonal onset of cracking. Then, as the crack progresses, Mode I becomes predominant and the failure surface aligns with the dominant longitudinal normal stresses due to bending. The geometrical symmetry midplane, which coincides with the loading symmetry plane, can be considered as an attractor of the twisting crack.

PolyMethyl MethAcrylate is an amorphous glass polymer which is characterized by homogeneous mechanical properties and brittle failure. The literature previously cited reports a Young's modulus $E=2800 \mathrm{MPa}$, a Poisson's ratio $\nu=0.38$, an elastic threshold uniaxial stress $\sigma_{y}=40 \mathrm{MPa}$ and fracture energy $G_{f}=500 \mathrm{~J} / \mathrm{m}$. Because of the clear role of tensile failure in this problem, it is natural to describe the inelastic processes using constitutive models based on Rankine's criterion.

In the finite element model, the beam is supported by two rollers on the lower surface which sustain only vertical forces (in the $\mathrm{Y}$ direction). The centerline on the top surface of the beam not only imposes a vertical displacement of $2 \mathrm{~mm}$, but also provides restraint to the out-of-plane forces ( $\mathrm{X}$ direction) and horizontal sliding ( $\mathrm{Z}$ direction). In the numerical analysis, the PMMA beam is subjected to monotonic loading.

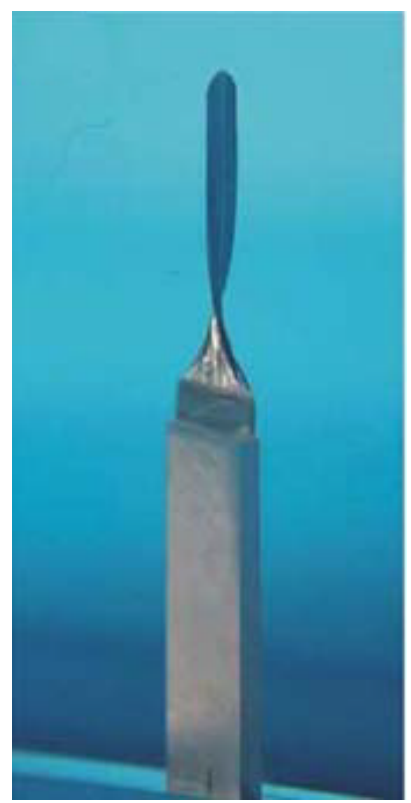

(a) Experiment

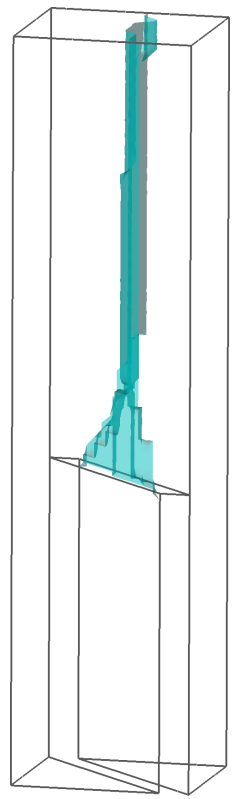

(b) IsoDamage

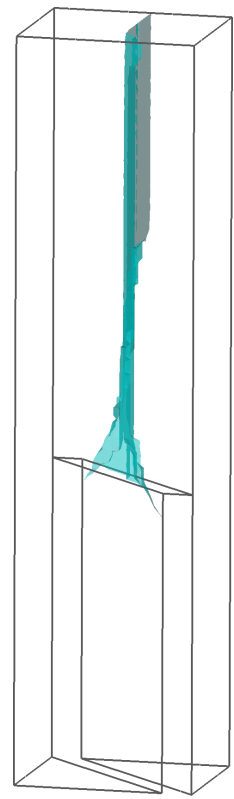

(c) Plasticity

Figure 6: Experimental [10] and numerical ( $\boldsymbol{\varepsilon}-\boldsymbol{u}$ formulation) crack surface of the three point bending test on PMMA. 


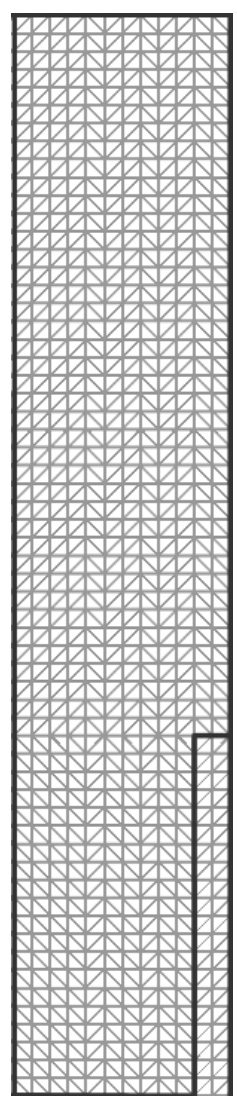

(a) Detail of the structured mesh in the middle region

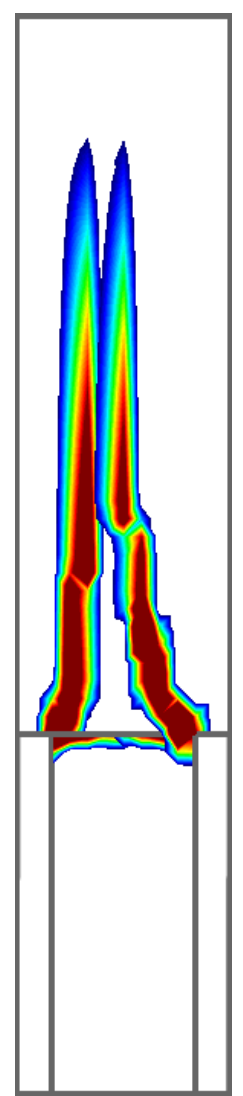

(b) $\boldsymbol{u}-\boldsymbol{\varepsilon}$ with IsoDamage

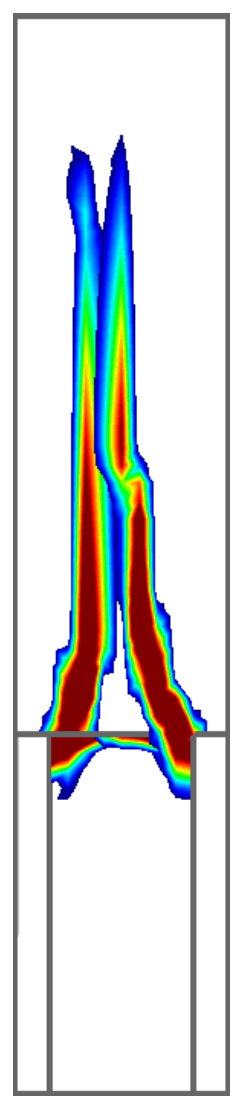

(c) $\boldsymbol{u}-\boldsymbol{\varepsilon}$ with Plasticity

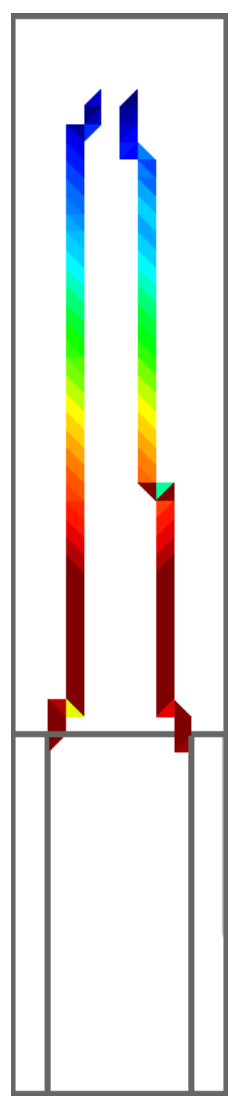

(d) $\boldsymbol{u}$ with IsoDamage

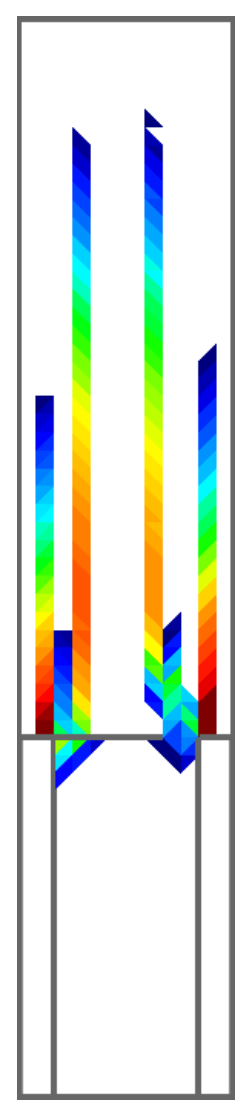

(e) $\boldsymbol{u}$ with Plasticity
EQ INEL STRAINS

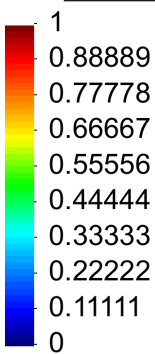

(f) Figure legend

Figure 7: Contour fills of major principal total strain in the crack at the front and back faces of the beam under 3 point bending test.

The FE mesh consists of tetrahedral elements (Figure 5 and Figure 7(a) for a detailed view), structured in the vicinity of the slot, where the elements have a characteristic size $h=1 \mathrm{~mm}$, and unstructured elsewhere. This allows to model the part subjected to localization with a $12 \times 10$ base grid and the notch is two elements wide. The grid of structure elements shows biased planes at $0^{\circ},+45^{\circ}, 90^{\circ}$ and $-45^{\circ}$. The final computational mesh is composed by 58,557 tetrahedral elements (11,677 points).

The objective of the numerical analysis is to test the mixed $\boldsymbol{\varepsilon}-\boldsymbol{u}$ formulation in comparison to the standard displacement-based FEM. This benchmark is challenging for two reasons. Firstly, the problem involves strain localization and crack propagation in a rather brittle material, which implies a sudden drop in stresses after cracking. As a consequence, global stability is sharply lost. Secondly, the discretization is rather coarse. In fact, from the notch to the midsection, the mesh presents just 4 elements; that is the available resolution to model the onset of failure propagation and its twisting and alignment with the mid-section.

Both Rankine-like isotropic damage and plasticity models provide similar solutions to this problem: Figure 6 compares the crack surface in the PMMA beam with the crack front propagating from the initial notch to the top surface, obtained with the two constitutive laws using the proposed mixed FE method. The numerical crack surfaces are plotted as the level set of zero horizontal displacements $(\mathrm{Z}$ direction), thanks to the symmetry of the problem.

Nevertheless, Figure 7 shows a detail of the mesh grid used for the computations and some substantial differences among the standard and mixed FE formulations in the contour fills of principal total strain in the crack, at the front and the back faces of the beam. Using the $\boldsymbol{\varepsilon}-\boldsymbol{u}$ formulation, the 


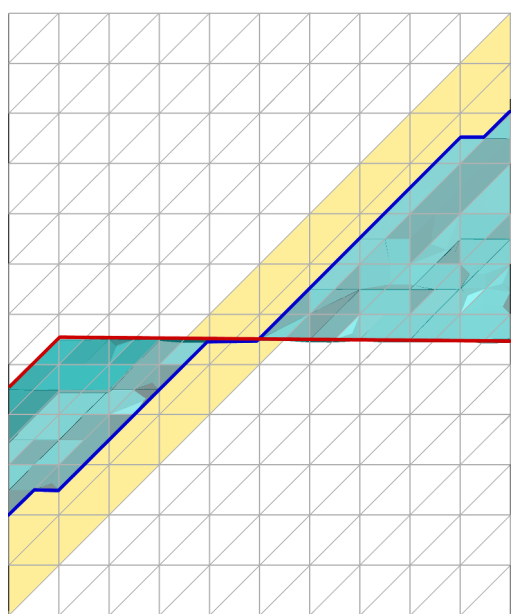

(a) $\boldsymbol{u}-\boldsymbol{\varepsilon}$ with IsoDamage

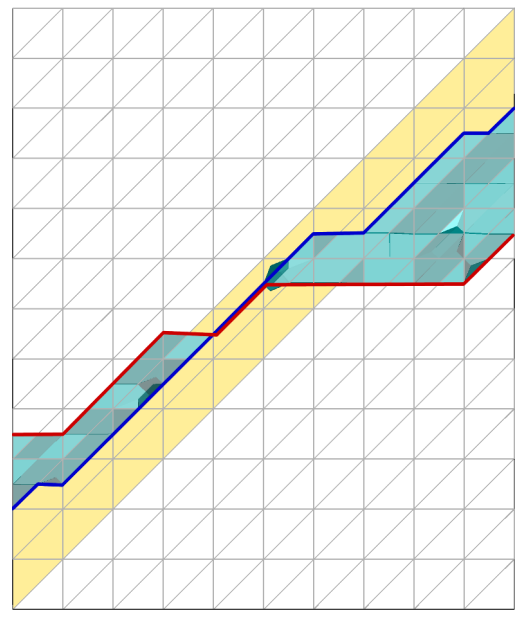

(c) $\boldsymbol{u}$ with IsoDamage

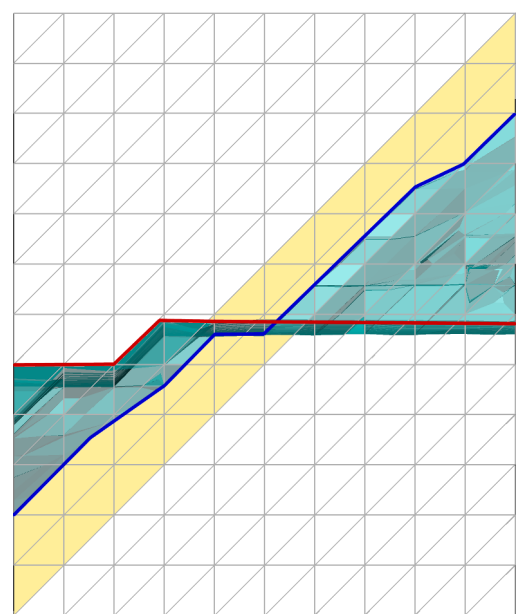

(b) $\boldsymbol{u}-\boldsymbol{\varepsilon}$ with Plasticity

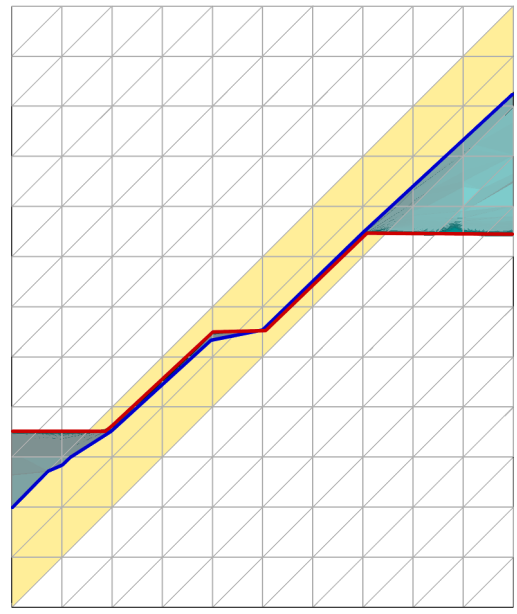

(d) $\boldsymbol{u}$ with Plasticity

Figure 8: Top view of the crack twist rotation in the 3 point bending test. Light yellow color represents the initial notch. Turquoise color represents the isosurface corresponding to the centerline of the localization band. The bottom crack profile is identified with the blue color while the top crack profile is highlighted in red.

failure surface is very similar with the two constitutive laws (Rankine isotropic damage and directional plasticity), with the strain softening band that initiate from two opposed notch corners and twists to the mid-section of the beam.

The solution given by the irreducible formulation is rather different. A substantially mesh-biased crack surface is obtained for both isotropic damage and plasticity cases: while the first one shows a slight tendency to converge to the center of the specimen, the latter one presents multiple vertical localization bands.

Owing to inter-element continuity of strains, mixed formulations for nonlinear analysis presents an effective localization band that spans two elements. The kinematic enhancement of using a continuous strain field results in a convergent and more accurate outcome than the standard finite elements, which suffer from severe mesh bias dependence.

In order to further compare the two FE technologies, Figure 8 shows the top view of the center line of the localization band, at a position $10 \mathrm{~mm}$ below the top surface. As discussed before, the crack starts from the $45^{\circ}$ slanted notch and then twists until it aligns with the mid-plane. Figure 8 depicts in yellow the initial notch profile and in turquoise the failure surface. In addition, the bottom crack profile is signaled in blue, whereas the top one is highlighted in red.

The initial profile of the localization band coincides in all four examples: the surface develops from 


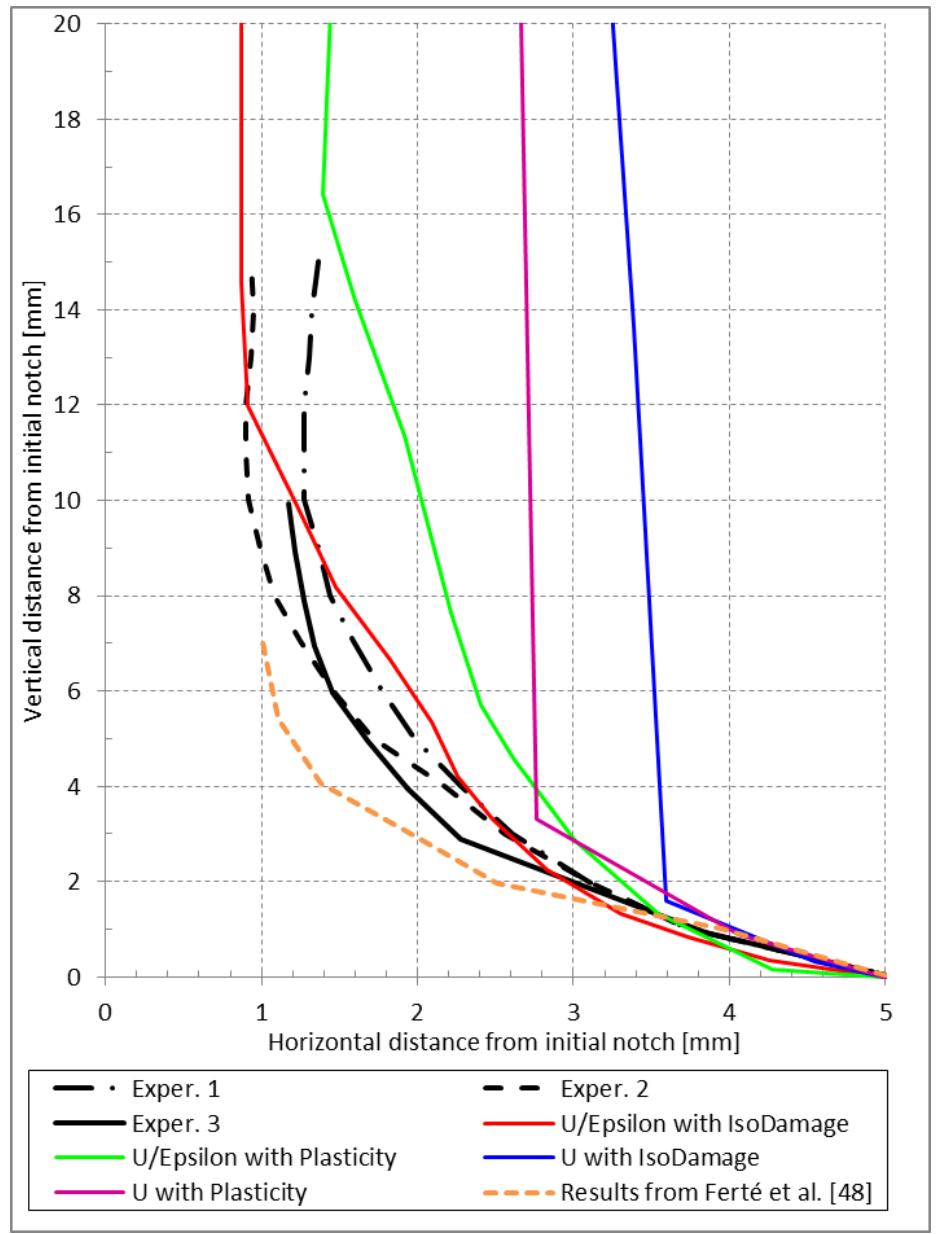

Figure 9: Plot of the computed crack path with respect to the experimental data from Citarella and Buchholz [47] and previous results from Ferté et al. [48].
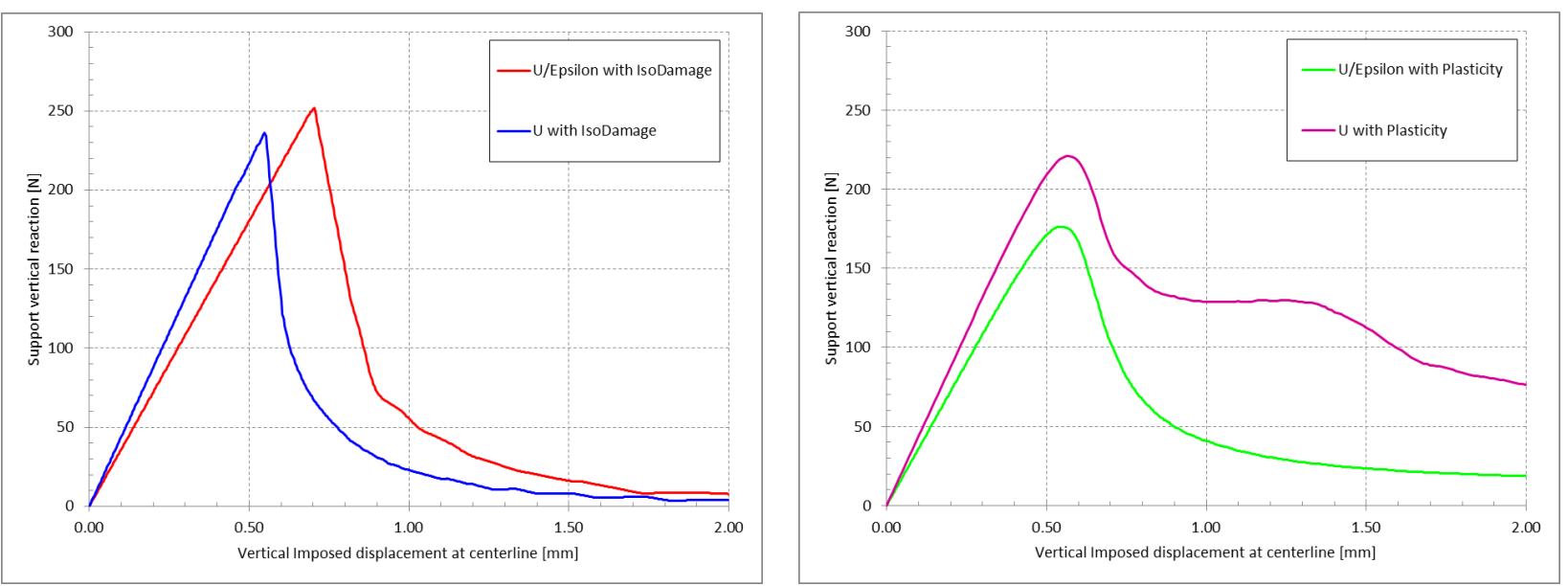

Figure 10: Plot of support reaction force with respect to the imposed vertical displacement. 
the two opposite inner corners of the notch and connects (almost) symmetrically at the center of the specimen. The mixed $\boldsymbol{\varepsilon}-\boldsymbol{u}$ formulation shows the expected twist rotation, with the final position of the crack surface close to the central symmetry plane of the beam. The slight asymmetry is due, on the one hand, to the orientation of the structured mesh and, on the other hand, to the use of a pure tensile failure criterion, which does not allow the crack surface to cross the compression head at the top of the beam. Concerning the irreducible formulation, the results shows a clear mesh bias, with a relatively small twisting rotation for the isotropic damage case and almost no twisting at all in the case of plasticity.

Figure 9 depicts the relative position of the computed crack path with respect to the notch location compared to the experimental results reported by Citarella and Buchholz [47] and the XFEM numerical simulation of Ferté et al. [48]. Once again, standard displacement based finite elements are unable to provide a satisfactory result, with the crack having a marked tendency to follow one of the directions of the mesh, independently from the constitutive law. Contrariwise, the mixed formulation achieves rather adequate outcomes. Both the isotropic damage and plasticity models converge at the top of the plot to the experimental range; in fact, the first one computes a crack path which is very close to the experiments. The only substantial differences are attributed to the relatively coarse mesh discretization in the vicinity of the notch.

Finally, the structural softening behavior is studied plotting the support reaction versus the imposed vertical displacement (Figure 10). The reduction of the carrying capacity is visible in all cases. In the elastic range, the standard and mixed finite elements show two different stiffnesses. For a given degree of refinement, the enhanced kinematics of the mixed formulation, with continuous strain fields, results in an accurate, and more flexible, response. Eventually, the two methods will converge to the same result in the elastic case as the mesh is refined.

The isotropic damage solution presents a well defined peak load with a sudden change of global behavior whereas the plastic one has a smoother transition. A similar sharp change in the carrying load was previously studied in PMMA specimens in the work of Cooke and Pollard [5]. The mixed FE is able to reproduce a monotonic softening branch with both constitutive laws. The displacementbased shows excessive energy dissipation in the plasticity case due to the multiple numbers of cracks developed during the inelastic branch of the test, see Figure $7(\mathrm{e})$.

\subsection{Prismatic skew notched concrete beam under torsion}

The second example is a prismatic skew edge notched beam under torsional load. This experiment was first performed by Jefferson et al. [49] on plain concrete specimens, but it was conducted on smaller PMMA beams as well by Buchholz et al. [50].

The test setup is shown in Figure 11: a prismatic beam with square base is positioned horizontally, with steel clamps at both ends. These present appendages on both sides which allow to avoid rigid movements and to apply the load. Three of the four steel arms are restrained in the vertical direction,

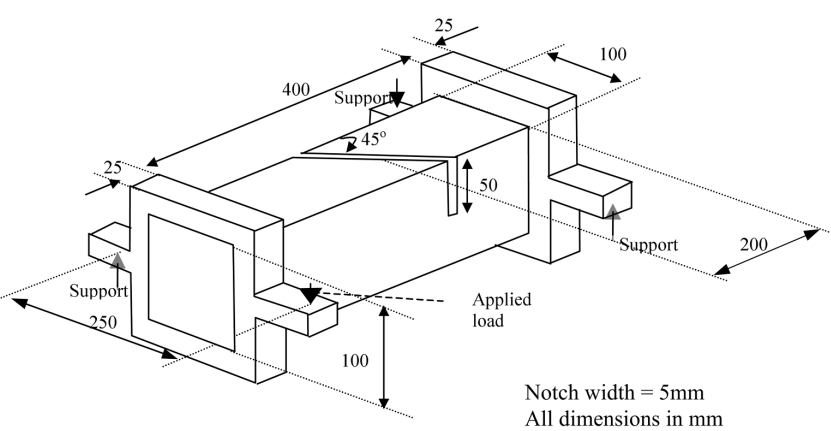

(a)

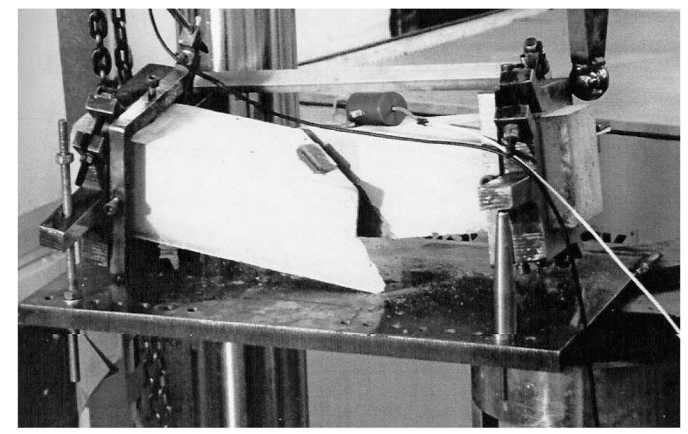

(b)

Figure 11: Geometry and experimental setup of the tests on prismatic beam under torsion with square cross section, from Jefferson et al. [49]. 


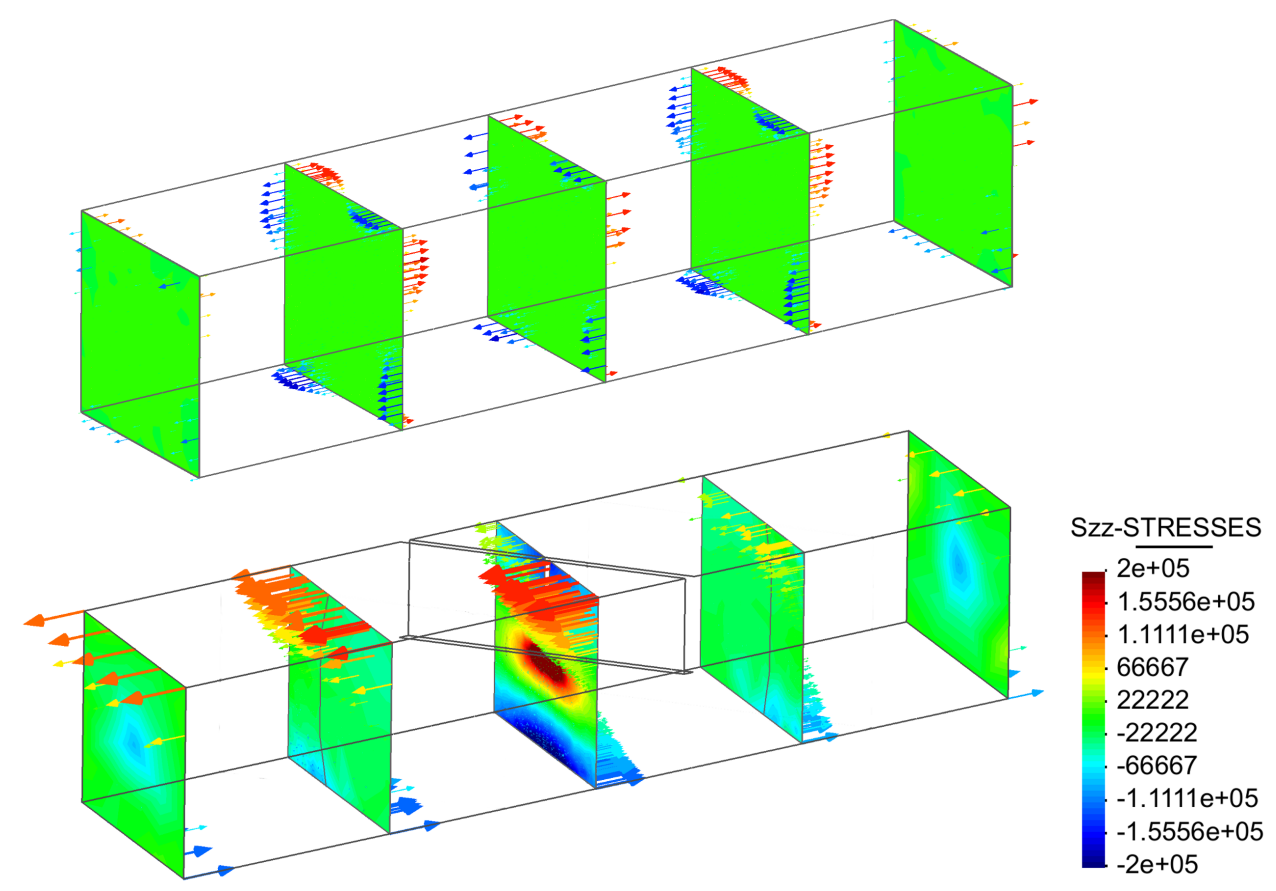

Figure 12: Plot of the contour fill of longitudinal stress distribution and vectors of longitudinal displacements due to uniform and non-uniform torsion on a prismatic square beam. Top figure shows the uniform (Saint Venant's) torsion of the unnotched specimen. Bottom figure shows the case of non uniform torsion of notched specimen.

whereas the last one is subjected to a concentrated load. The clamping frame is assumed to make perfect contact with the concrete specimen and ensure the transferring of the eccentric load to the specimen, resulting in a torsional moment aligned with the axis of the beam. It is also designed not to constrain warping of the end cross-sections.

The objective of this experiment is to test the tensile strength of plain concrete under torsion. The highest stress is located in the vicinity of the notch, with maximum values on the lateral surface of the beam. According to several works in the field $[1,6,4]$, the fracture initiation is caused by a mode III loading with transition to mode I brittle failure.

An unnotched specimen would be subjected to uniform (Saint-Venant's) torsion, characterized by a uniform warping of the cross section, unhindered by the design of the clamping frames at both ends. Under uniform torsion, no longitudinal normal stress $\left(\boldsymbol{\sigma}_{z z}\right)$ would develop.

The slanted notch induces non-uniform torsion. This is shown in Figure 12(b), which overlaps contour fills of the longitudinal normal stresses $\left(\boldsymbol{\sigma}_{z z}\right)$ and vectors of longitudinal (warping) displacement at five different cross-sections of the beam, in the elastic regime. It is obvious that: (i) the warping displacement is not uniform, being rather distorted by the notch and (ii) the $\boldsymbol{\sigma}_{z z}$ stresses are not zero, particularly in the vicinity of the notch. Note that these non-vanishing normal stresses have null resultant axial force and null resultant bending moments, but they have a resultant warping moment, also known as bimoment. In comparison, Figure 12(a) depicts the case for the unnotched specimen, where uniform displacement and null stress are observed.

Considering tensile fracture as the main cause for cracking of unreinforced concrete, this example investigates the differences between the isotropic damage and plasticity constitutive laws, both based on Rankine's failure criterion with the same mechanical parameters. The material properties are (from [49]): Young's modulus $E=35 \mathrm{GPa}$, Poisson's ratio $\nu=0.2$, tensile uniaxial strength $\sigma_{y}=2.3 \mathrm{MPa}$ and fracture energy $G_{f}=80 \mathrm{~N} / \mathrm{m}$.

The mesh is composed by 67,038 tetrahedral elements $(12,729$ nodes) subdivided in three regions. The part of the beam around the notch consists of $\boldsymbol{\varepsilon}-\boldsymbol{u}$ mixed finite elements with nonlinear constitutive behavior; outside this, the remaining concrete volume in contact with the clamps and, finally, 
the steel frame itself are elastic. In the latter two regions, the FEM formulation is the standard displacement-based, since no cracking is expected there.

The boundary conditions, as demonstrated in [35], are crucial for the correct numerical analysis of the test. In the present case, the model constraints are recovered from photos to be as close as possible to the experimental setup. From Figure 11(b), details of the boundary conditions can be extracted. Three vertical supports consist of steel posts, allowing for free rotation. Two of them (the closest and furthest ones in the photo) restrain the horizontal motion of the beam. Finally, the free appendage is subjected to an imposed vertical displacement.

In the numerical model, the four appendages are restrained vertically in a single point. Taking advantage of the symmetry of the setup, the horizontal motion is limited by constraining the center of the front and rear faces of the beam. Both the geometry and the loading conditions are skewsymmetric, i.e. a $180^{\circ}$ rotation of the problem would result in the same test setup. Given the nature of the problem, localization of strains is expected to be skew-symmetric.

The results are presented and discussed next. The displacement solution at the end of the test, with the corresponding deformation, is presented in Figure 13. Here, similar solution fields are captured for the two constitutive laws: the opening of the notch allows for a substantial rotation of one beam end from the other, meaning that localization of strain has occurred.

Figure 14 compares the experimental crack surface with the numerically computed ones, at the end of the analysis. While both plasticity and damage constitutive laws are able to predict correctly the global behavior, it is possible to notice that the latter one provides a more curved profile than the first one. In the case of damage, the crack starts from the notch almost horizontally and, then, rotates to reach the expected angle of $45^{\circ}$. On the contrary, in the case of plasticity, the propagation direction of the crack is constantly orientated at $45^{\circ}$.

The two solutions can be also compared with the top view of the experimental tests on PMMA, as depicted in Figure 15, and a side perspective in Figure 16. In all cases, a skew-symmetric crack is obtained as expected. The top view shows that both material laws, used with the mixed straindisplacement finite elements, are able to describe effectively the complex twisting crack pattern.

The peculiar arch-like shape, observable in the side view, proves that the highest tensile stress is located on the external surface of the beam, as previously discussed. Contrariwise, the central part is under compressive stresses arising from the additional bending. In the numerical analyses, as the failure criterion only involves the major principal tensile stress, the bottom part of the beam remains elastic.

Finally, Figure 17 shows the force-displacement plots. The experimental data is compared with the numerical solution using the $\varepsilon-\boldsymbol{u}$ formulation with isotropic damage and plasticity. There is a

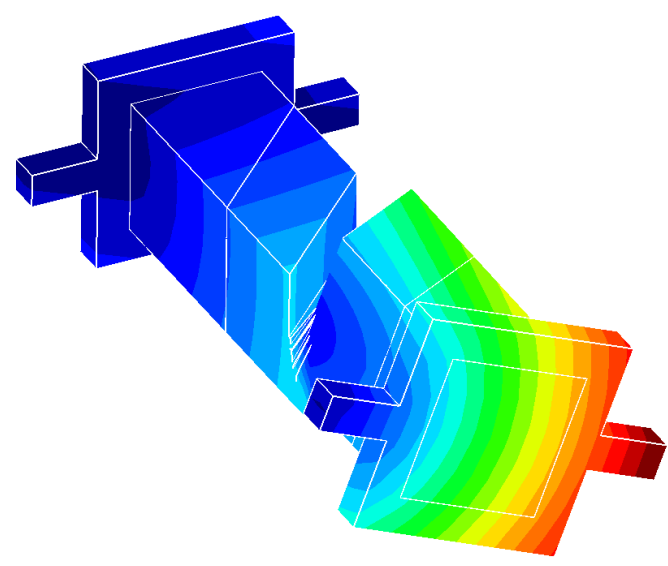

(a) Isodamage

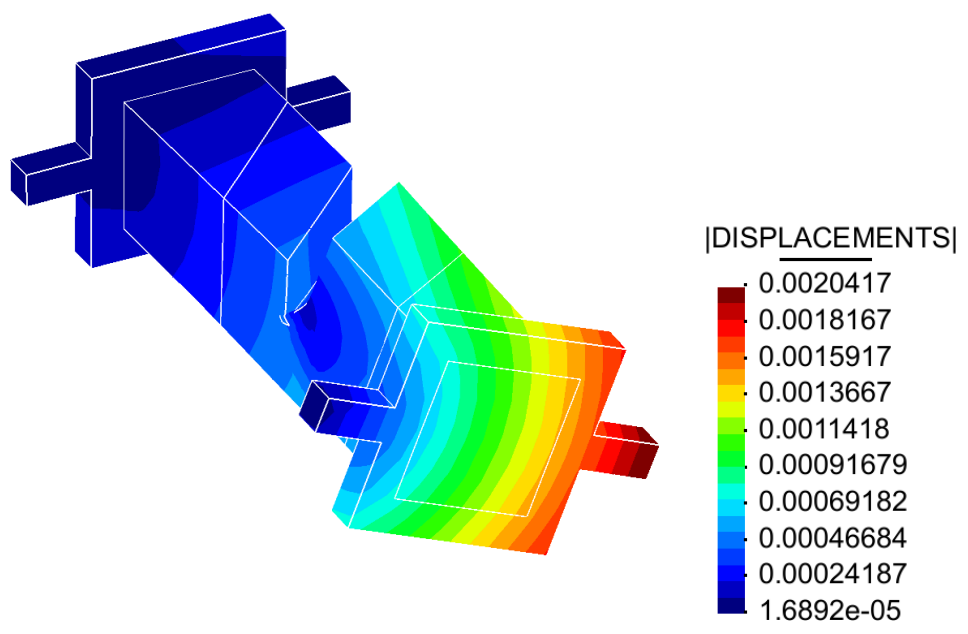

(b) Plasticity

Figure 13: Plot of the contour fills of the displacement field superposed on the computed beam deformation at the end of the test. The isotropic damage solution is very similar to the plasticity one. 


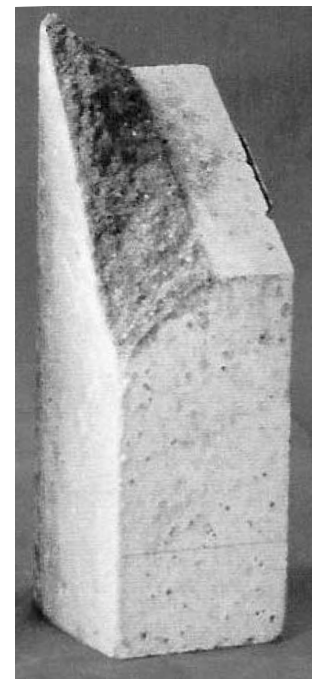

(a)

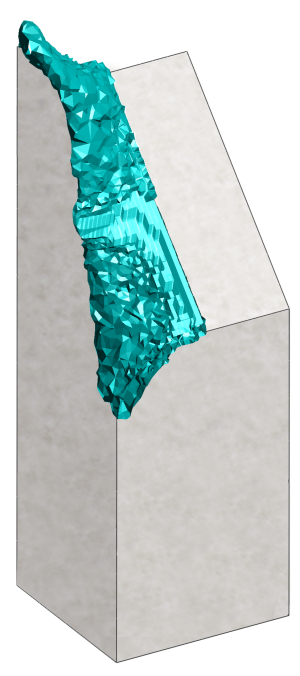

(b)

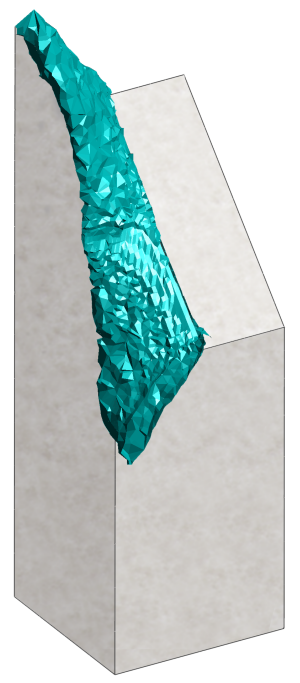

(c)

Figure 14: Comparison of (a) the experimental outcome with the computed crack surfaces in the case of (b) isotropic damage and (c) plasticity for the prismatic beam with square cross section.

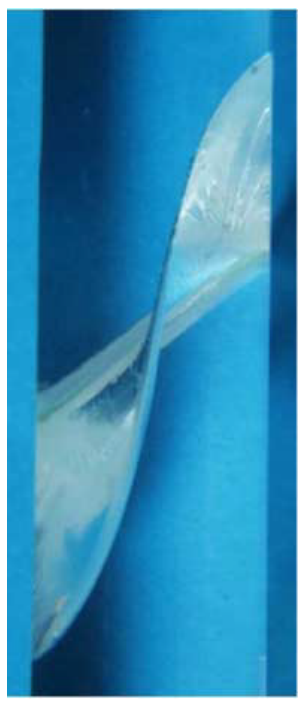

(a)

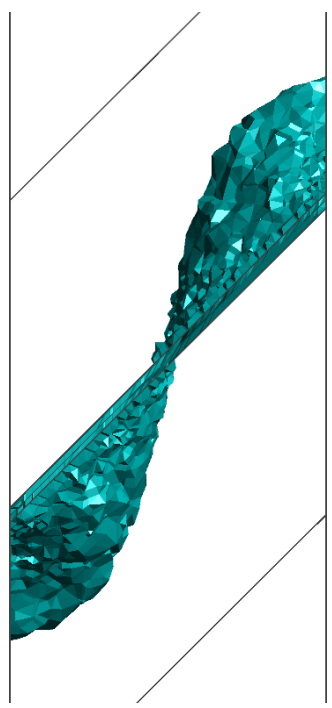

(b)

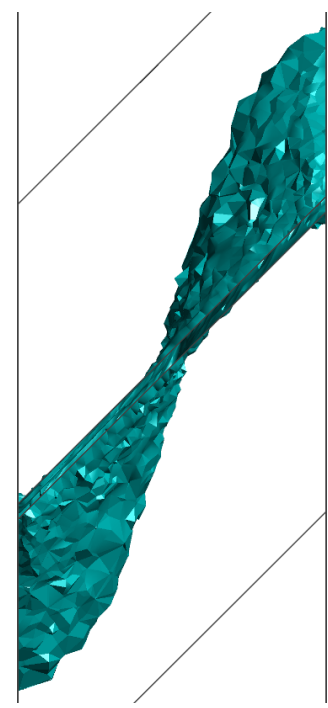

(c)

Figure 15: Top views of the crack surface from (a) tests on PMMA [50], (b) Isotropic Damage and (c) Plasticity.

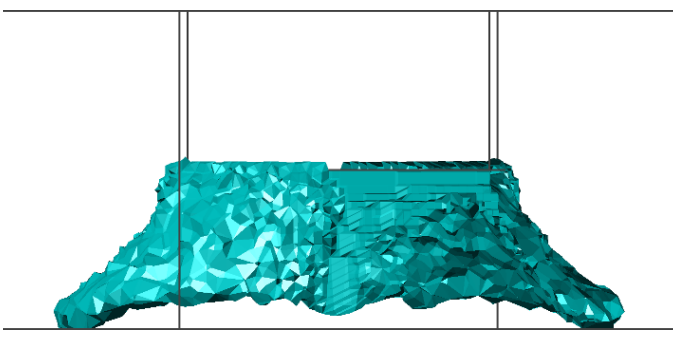

(a)

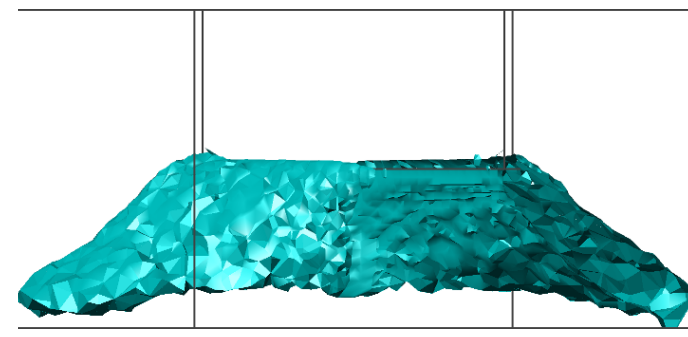

(b)

Figure 16: Side view of the fracture surface: (a) Isotropic Damage and (b) Plasticity. 


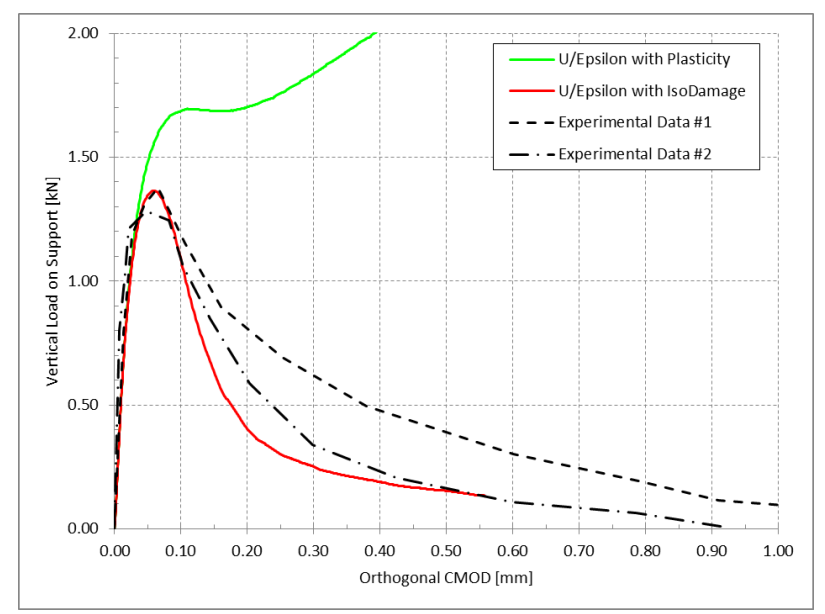

(a)

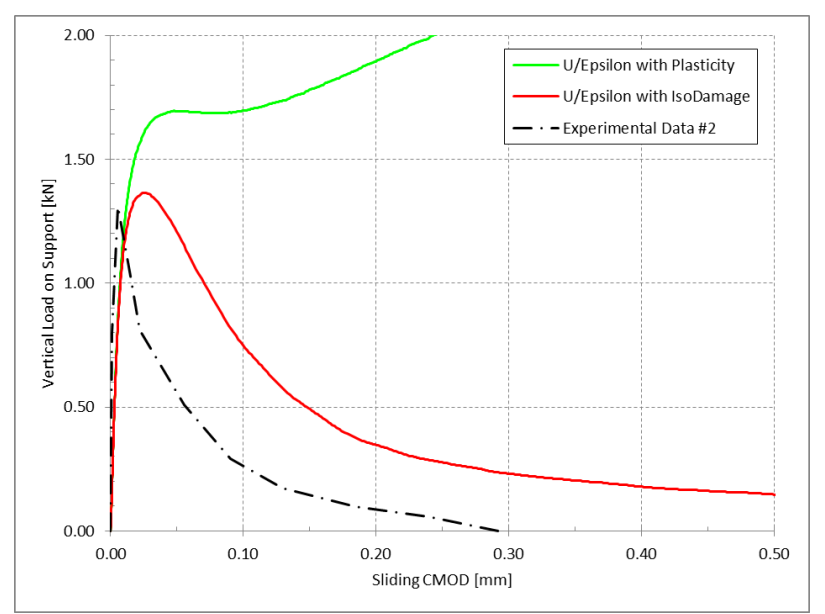

(b)

Figure 17: Plots of vertical force versus (a) orthogonal CMOD and (b) sliding CMOD

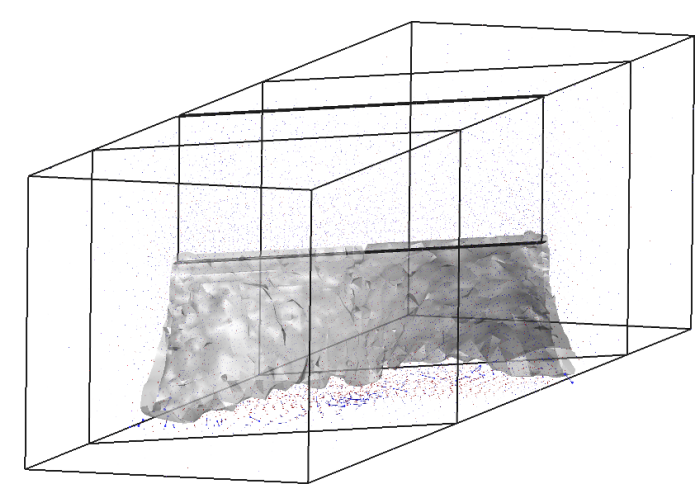

(a)

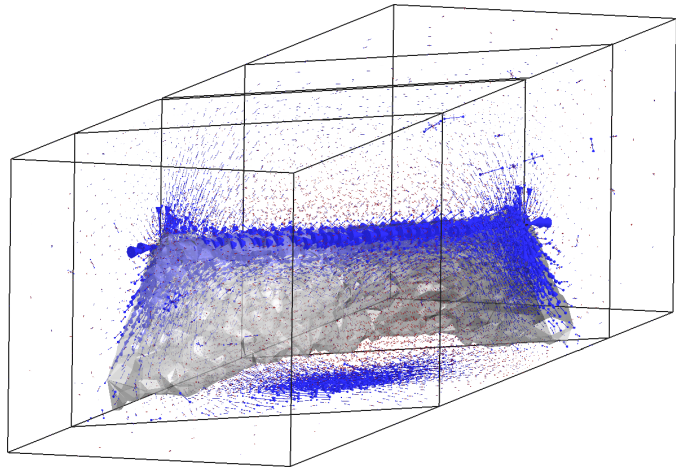

(b)

Figure 18: Residual principal stresses in the (a) isotropic Damage and (b) plasticity cases. Blue vectors represents compressive stress, red vectors represents tensile stress.

major difference in the global behavior: the case involving damage shows an almost complete loss of load carrying capacity due to strain softening whereas the plasticity one does not. Instead, the plastic model reaches a plateau and, then, the load carrying capacity increases again.

Indeed, there is a fundamental difference in the definition of the plastic and damage constitutive laws. Plasticity is based on the definition of plastic flow, which gives a directional character to the inelastic deformation. Moreover, associative plasticity implies that the stress reduction is proportional to the plastic flow, but also to the elastic constitutive tensor; hence, it is affected by the Poisson's effect. Such orthotropic behavior does not ensure a complete stress release even in softening cases. In fact, Poisson's effect generate significant residual compressive stresses around the crack associated to the localization of the opening strains, as it is shown in Figure 18(b). This stress field is restrained by the longitudinal boundary conditions and, consequently, it increases the force required for the progression of the localization.

In contrast, isotropic damage reduces the material stiffness isotropically, resulting in a uniform reduction of all the principal stresses. In Figure 18(a) stresses are almost completely released. The only remaining stresses are the compressive ones due to the non uniform torsion caused by the slanted notch.

\subsection{Cylindrical skew notched concrete beam under torsion}

In this final example, the test on a skew-notched cylindrical plain concrete beam subjected to torsion is modeled. The experimental setup is quite similar to the previous example. Figure 19(a) shows 


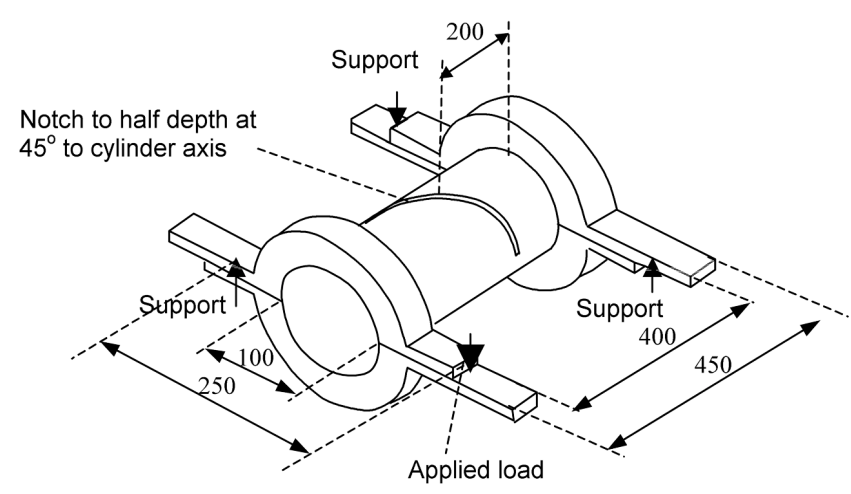

(a)

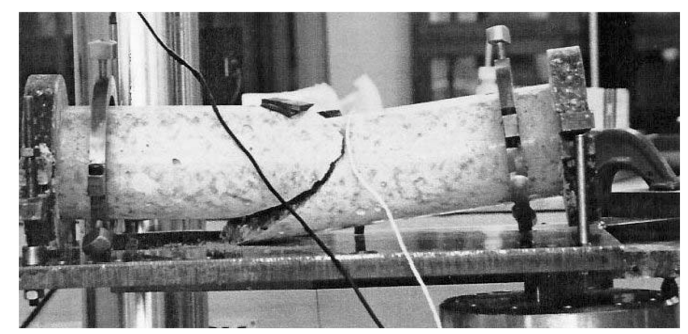

(b)

Figure 19: Geometry and experimental setup of the tests on cylindrical beam under torsion, from Jefferson et al. [49].

the characteristic dimensions of the specimen and of the steel frame. The cylindrical beam has the same length as the prismatic one and also presents a $45^{\circ}$ notch located at the center of the specimen. The clamping system is visible in Figure 19(b) and, likewise, it provides the transfer of external vertical imposed displacement to the specimen as a torsion force and ensures proper restraint of rigid movements. Therefore, boundary conditions are identical to the ones used in the previous example.

The material properties are assumed the same as in the last example: Young's modulus $E=35$ $\mathrm{GPa}$, Poisson's ratio $\nu=0.2$, tensile uniaxial strength $\sigma_{y}=2.3 \mathrm{MPa}$ and fracture energy $G_{f}=80$ $\mathrm{N} / \mathrm{m}$. Indeed, the referenced work by Jefferson et al. [49] is followed, although the batch of concrete for the cylindrical beams has not been tested for mechanical parameters. The numerical analysis has been performed considering Rankine's and Drucker-Prager's (with $45^{\circ}$ friction angle) failure criteria. In fact, the objective of this example is to assess the influence of the shear stresses in the experimental results and the failure mechanism.

Concrete is frequently thought to fracture in mode I and Rankine based criteria have been widely adopted. However, when dealing with mode III and mixed mode loading, the dependence of shear strength from pressure can play a fundamental role in the prediction of the failure mechanism. Consequently, it is convenient to introduce a pressure dependent model such as Drucker-Prager. Note that, as shown in Saloustros et al. [51], Rankine's criterion is a limit case of a family of pressure dependent constitutive models.

The mesh consists of 62,309 tetrahedral elements (11,892 nodes) which concentrate in the vicinity of the notch. In order to save on computational resources, the central part of the FE mesh is modeled using mixed $\boldsymbol{\varepsilon}-\boldsymbol{u}$ finite elements whereas the two beam ends as well as the steel frame are modeled using irreducible displacement-based elements, since, in these regions, inelastic phenomena do not appear.

An unnotched circular beam, subjected to uniform (Coulomb's) torsion, does not present any warping nor longitudinal stresses. Figure 20(a) shows the null field of $\boldsymbol{\sigma}_{z z}$ and null warping displacements in the elastic range at five different cross sections. As in the case of the square prismatic beam, the slanted notch causes the torsion to be non uniform, resulting in nonzero stresses $\boldsymbol{\sigma}_{z z}$ and longitudinal warping displacements, with the maximum values in the vicinity of the cut (see Figure 20(b)).

Figure 21 compares the final shape of the crack surface for the four analyzed cases. No images of the experimental crack surface are given in [49]. Plasticity and isotropic damage in the case of Rankine's failure criterion show a larger and more complex fracture geometry, whereas the Drucker-Prager's ones are sensibly closer to the notch cross section. The top views of such surfaces are presented on Figure 22 and the side perspectives are depicted in Figure 23. Rankine's cases show a behavior similar to the square prismatic beam, with a typical skew-symmetric curved shape; the circular shape of the specimen causes a more curved bottom profile of the failure surface.

Similarly, the Drucker-Prager criterion shows a curved profile, although rather smaller. The less pronounced crack surface is the direct result of a failure criterion that is based on the interdependence 


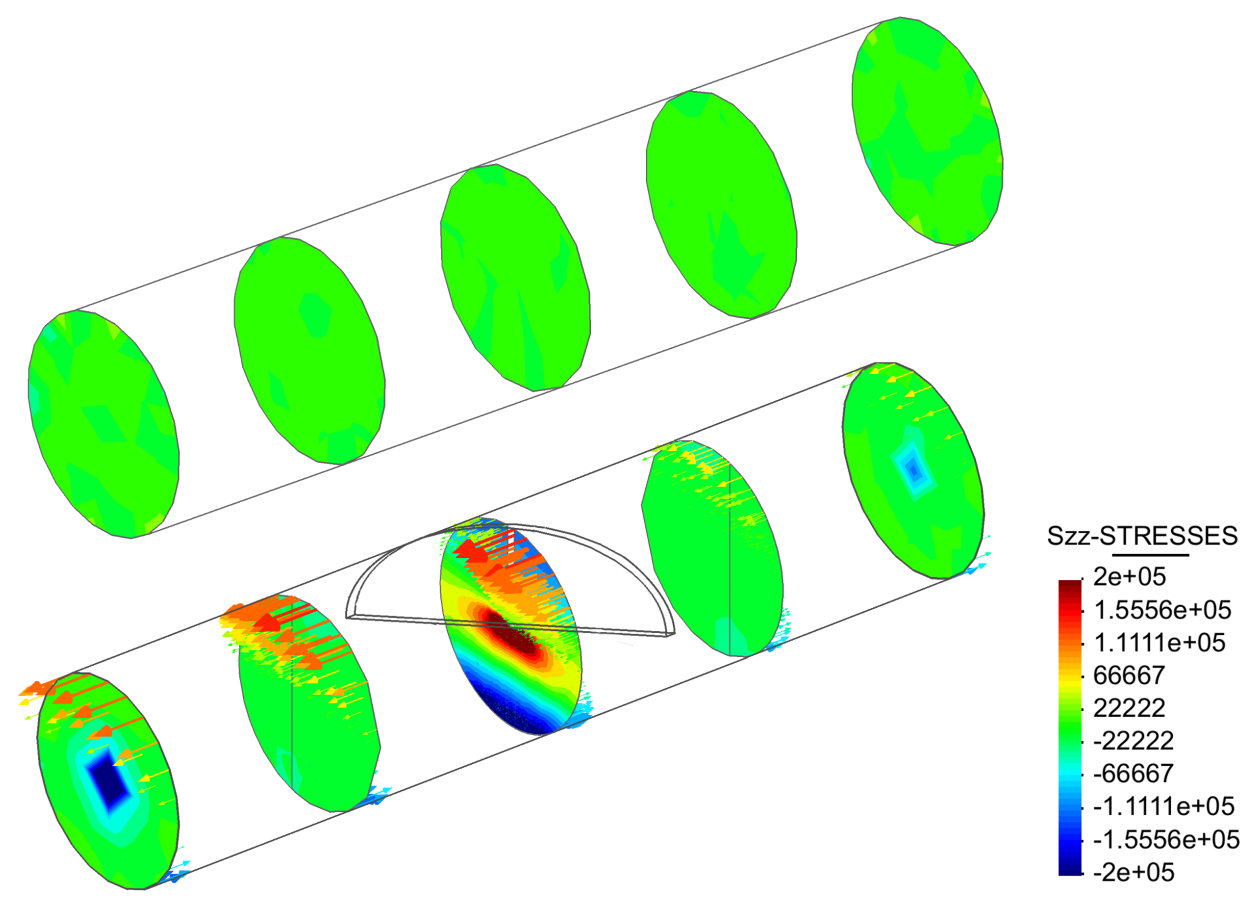

Figure 20: Plot of the contour fill of longitudinal stress distribution and vectors of longitudinal displacements due to uniform and non-uniform torsion on a cylindrical beam. Top figure shows the uniform (Coulombs's) torsion of the unnotched specimen. Bottom figure shows the case of non uniform torsion of notched specimen.

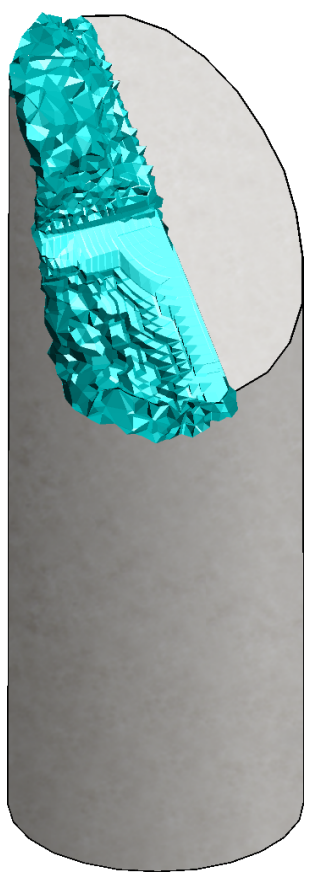

(a)

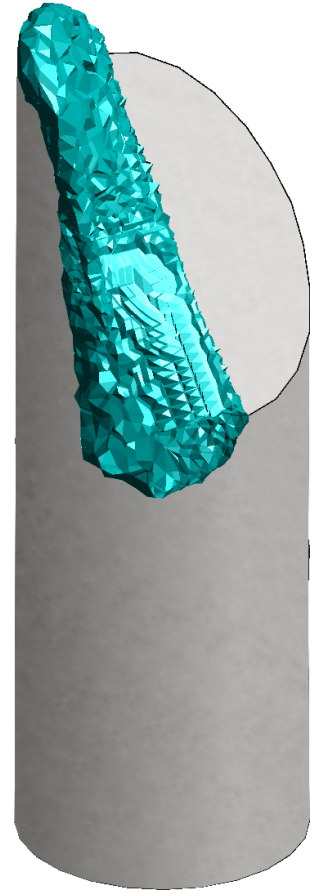

(b)

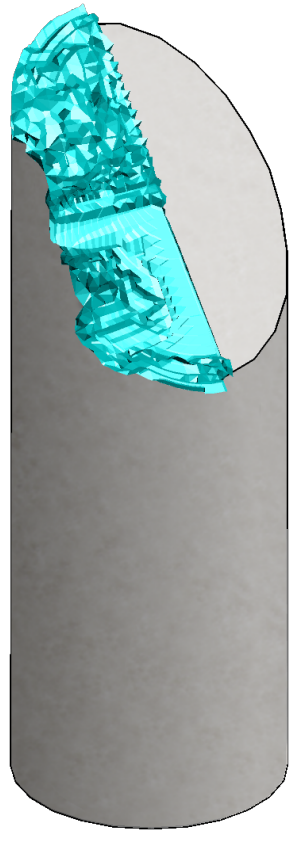

(c)

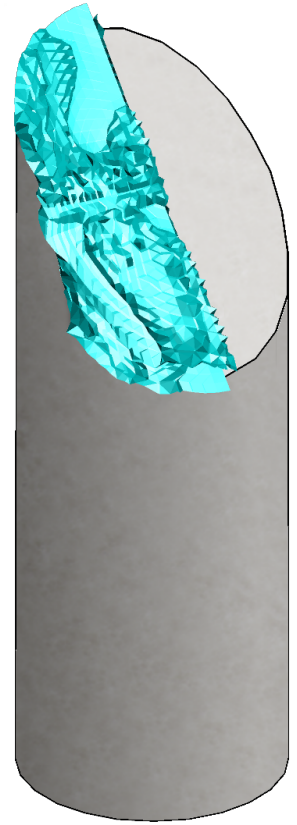

(d)

Figure 21: View of the crack surface at the end of the analysis from (a) Rankine Isotropic Damage, (b) Rankine Plasticity, (c) Drucker-Prager isotropic damage and (d) Drucker-Prager plasticity solutions. 


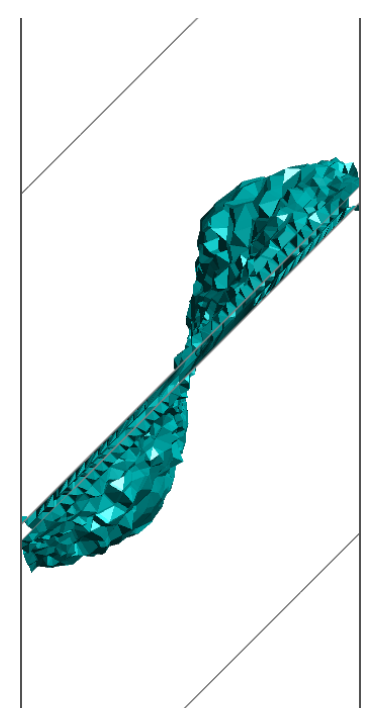

(a)

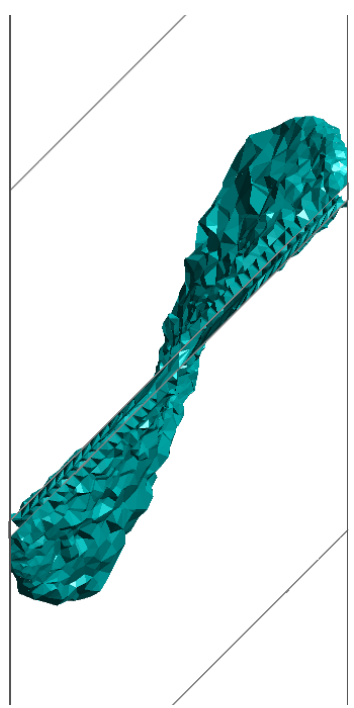

(b)

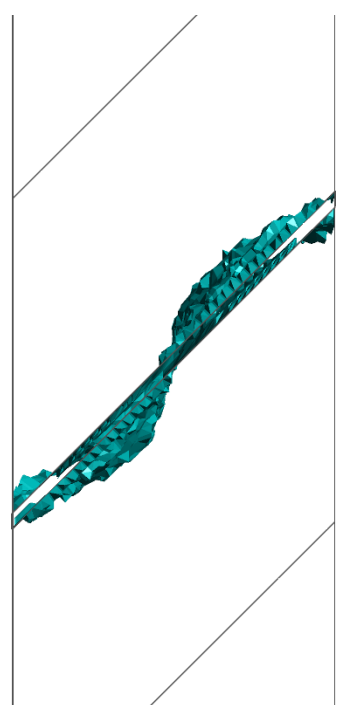

(c)

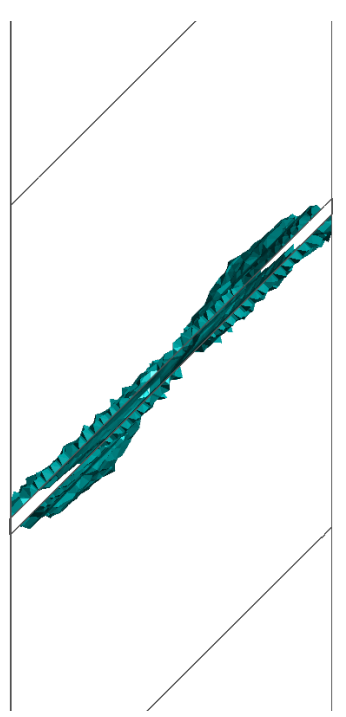

(d)

Figure 22: Top view of the crack pattern from (a) Rankine Isotropic Damage, (b) Rankine Plasticity, (c) Drucker-Prager isotropic damage and (d) Drucker-Prager plasticity solutions.

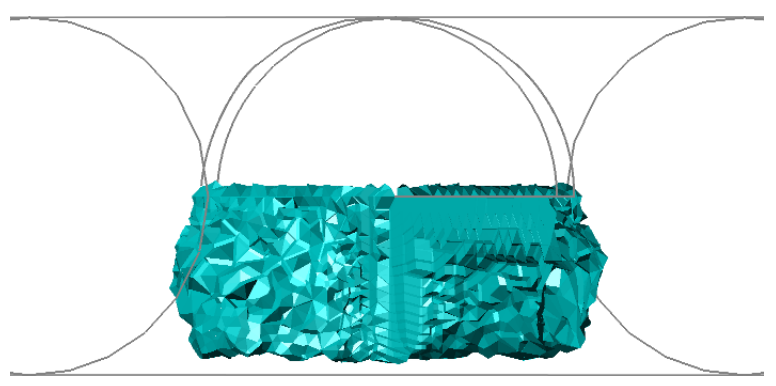

(a)

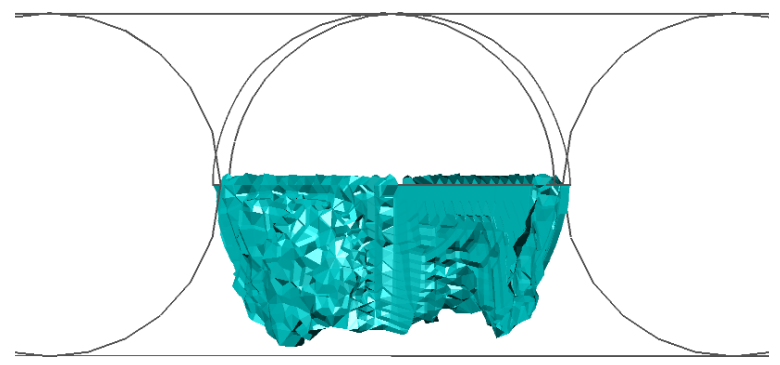

(c)

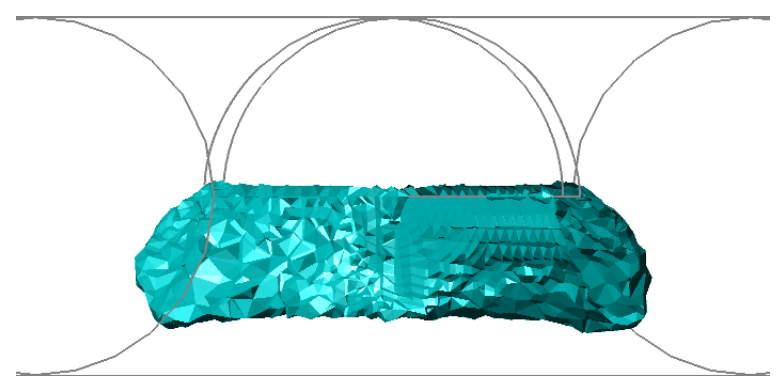

(b)

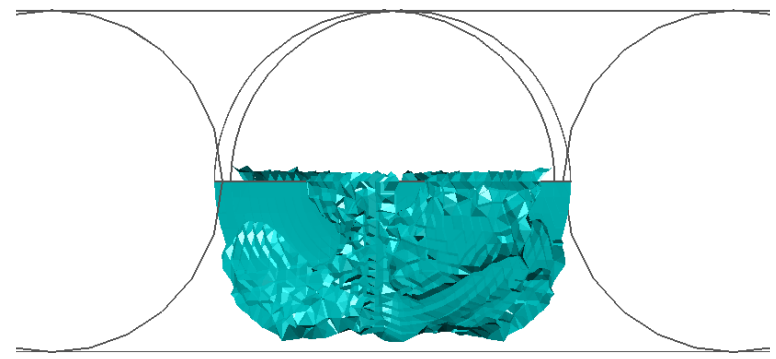

(d)

Figure 23: Side view of the crack pattern from (a) Rankine Isotropic Damage, (b) Rankine Plasticity, (c) Drucker-Prager isotropic damage and (d) Drucker-Prager plasticity solutions. 


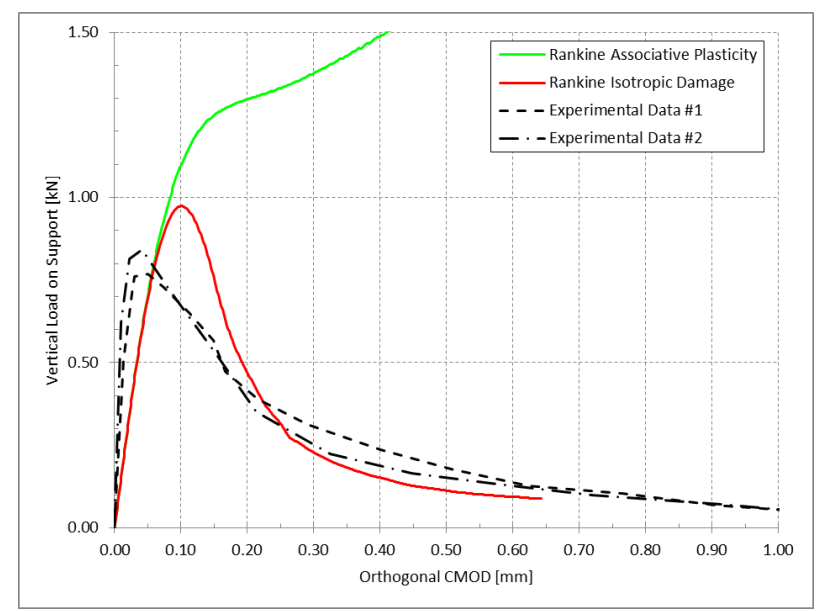

(a)

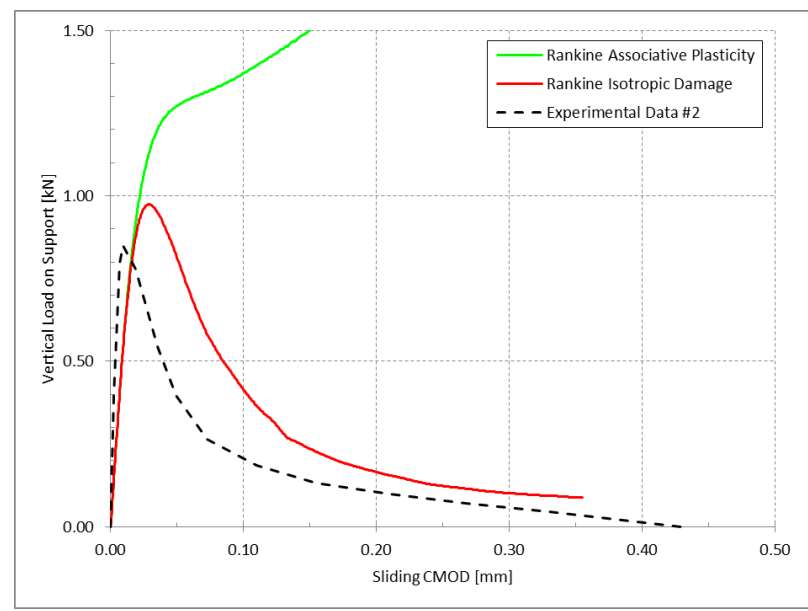

(b)

Figure 24: Plots of vertical force versus (a) orthogonal CMOD and (b) sliding CMOD using Rankine failure criterion

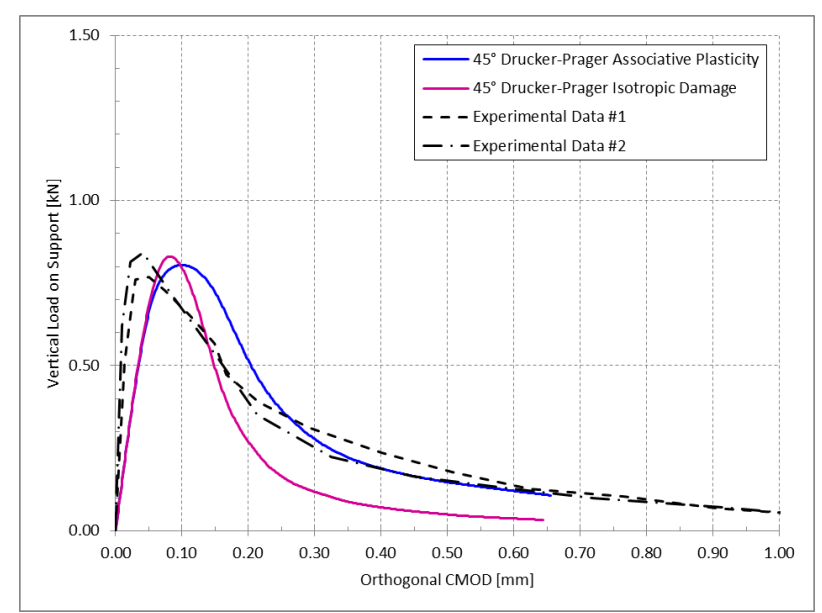

(a)

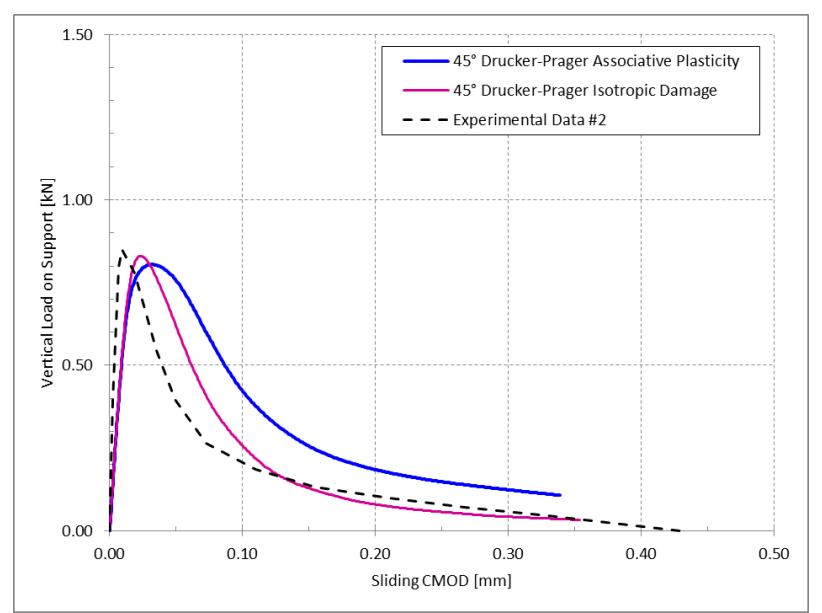

(b)

Figure 25: Plots of vertical force versus (a) orthogonal CMOD and (b) sliding CMOD using DruckerPrager failure criterion with $45^{\circ}$ friction angle

of pressure and shear through the friction angle, in lieu of the major principal stress.

Finally, Figure 24 shows the plot of applied load versus orthogonal and sliding CMOD values with the Rankine constitutive law while Figure 25 shows the same plot for the Drucker-Prager case. Here, the experimental values from [49] are compared with the numerical analyses.

In the first place, it is observed that the stiffness of the experimental specimen differs substantially from that of the numerical analyses. Since it is reported that a different batch was used for the cylinder specimens, it is possible that the concrete mix might have had a higher Young's modulus.

Notwithstanding, the numerical analyses with the Drucker-Prager failure criterion predict the peak load satisfactorily, followed by full reduction of stresses. Contrariwise, the Rankine based models provide different results. As in the previous example, plasticity fails to produce decrease of loadcarrying capacity while isotropic damage presents a reduction of the total load, but the peak load value is slightly overestimated.

Hence, the numerical analysis shows that the torsional tests on circular specimens require a detailed description of the mixed mode failure of concrete. On the one hand, the numerically computed fracture surface shows a similar "S" shaped profile in both cases. On the other hand, the softening behavior appears to be properly captured by the $\boldsymbol{\varepsilon}-\boldsymbol{u}$ mixed finite elements when using Drucker-Prager's model with $45^{\circ}$ friction angle whereas Rankine's one slightly overestimates the peak load. 


\section{Computational pay-off of kinematic compatibility and iterative procedure}

The computational time and memory requirements for the last two examples are presented in the following tables. Three different finite element technologies are considered: full mixed $\boldsymbol{\varepsilon}-\boldsymbol{u}$, combined kinematically compatible mixed and irreducible FE and full displacement-based standard $\boldsymbol{u}$ formulations. In the first one, the whole mesh consists of mixed elements and, for this reason, it is the most demanding from the computational point of view. The second one combines compatible elements in the same mesh; its performance pay-off will be benchmarked. Finally, the third one, with the standard irreducible only, is the less demanding.

These numerical analysis are run on a desktop computer with 8 GB of RAM and a dual core CPU clocking at $2.83 \mathrm{GHz}$.

The prismatic skew notched concrete beam under torsion is calculated with a mesh of 67,038 elements. When the combined formulation is used, the computational grid is composed of 9,783 irreducible and 57,255 mixed finite elements. Table 2 shows a substantial reduction in computational time for the proposed methodology. Similarly, the RAM usage is slightly reduced. The irreducible formulation is added for reference, as the corresponding results are generally deficient.

\begin{tabular}{|l|c|c|c|c|c|c|}
\hline Formulation & Solver & $\begin{array}{c}\text { CPU } \\
t_{\text {fact }}(\mathrm{s})\end{array}$ & $n_{\text {iter }}$ & $\begin{array}{c}\text { CPU } \\
t_{\text {iter }}(\mathrm{s})\end{array}$ & $\begin{array}{c}\text { CPU } \\
t_{\text {step }}(\mathrm{s})\end{array}$ & RAM (MB) \\
\hline Full mixed $\boldsymbol{\varepsilon}-\boldsymbol{u}$ formulation & Newton-Raphson & 71 & 3 & 71 & 213 & 5660 \\
\cline { 2 - 7 }$(67,038$ elements $)$ & Secant scheme & 55 & 10 & 2 & 75 & 2833 \\
\hline Mixed $\boldsymbol{\varepsilon}-\boldsymbol{u}$ and irreducible $\boldsymbol{u}$ & Newton-Raphson & 61 & 3 & 61 & 183 & 4129 \\
\cline { 2 - 7 }$(57,255+9,783$ elements) & Secant scheme & 51 & 10 & 2 & 71 & 2065 \\
\hline Irreducible $\boldsymbol{u}$ & Newton-Raphson & 7 & 3 & 7 & 21 & 683 \\
\cline { 2 - 7 }$(67,038$ elements $)$ & Secant scheme & 6 & 10 & 2 & 26 & 369 \\
\hline
\end{tabular}

Table 2: CPU time and RAM memory requirements in the prismatic skew notched concrete beam under torsion. The proposed method is compared with the full mixed and full irreducible formulations. Likewise, the Newton-Raphson and Secant schemes are compared per first iteration factorization time, number of iterations and step average time.

The cylindrical skew notched concrete beam under torsion is modeled with 62,309 elements. In the case of combination of kinematically compatible FE, the mesh is subdivided in 53,876 mixed and 8,433 irreducible elements. CPU time gains are similar to the ones observed for the prismatic beam case.

Note that using the irreducible formulation in only $14 \%$ of the total number of elements translates directly in a $14 \%$ pay-off of CPU time per step in the Newton-Raphson, and $5.6 \%$ in the Secant method. In analyses where the ratio of irreducible to mixed elements can be greater, the gain increases correspondingly.

Moreover, for each case, the performance of the Newton-Raphson solver is compared with the Secant scheme. The first one requires a lower number of iterations per step thanks to the quadratic convergence given by the consistently linearized global matrix. Nevertheless, each iteration requires the solution of the full updated algebraic system which, as in this case, can not be symmetric.

Contrariwise, the modified Secant scheme updates the global matrix only at the beginning of each step and then it iterates using the already factorized system. Although the rate of convergence is linear and more iterations are needed, it results in a faster procedure. In both of the proposed examples, the computational time for the secant solver is less than half of the Newton-Raphson for the same convergence tolerance, which is set to $10^{-3}$ with respect to the residual forces. Moreover, the symmetry of the matrix reduces the required memory to almost half. 


\begin{tabular}{|l|c|c|c|c|c|c|}
\hline Formulation & Solver & $\begin{array}{c}\text { CPU } \\
t_{\text {fact }}(\mathrm{s})\end{array}$ & $n_{\text {iter }}$ & $\begin{array}{c}\text { CPU } \\
t_{\text {iter }}(\mathrm{s})\end{array}$ & $\begin{array}{c}\text { CPU } \\
t_{\text {step }}(\mathrm{s})\end{array}$ & RAM (MB) \\
\hline Full mixed $\boldsymbol{\varepsilon}-\boldsymbol{u}$ formulation & Newton-Raphson & 65 & 3 & 65 & 195 & 4459 \\
\cline { 2 - 7 }$(62,309$ elements) & Secant scheme & 50 & 8 & 2 & 66 & 2232 \\
\hline Mixed $\boldsymbol{\varepsilon}-\boldsymbol{u}$ and Irreducible $\boldsymbol{u}$ & Newton-Raphson & 54 & 3 & 54 & 162 & 3334 \\
\cline { 2 - 7 }$(53,876+8,433$ elements $)$ & Secant scheme & 45 & 8 & 2 & 61 & 1668 \\
\hline Irreducible $\boldsymbol{u}$ & Newton-Raphson & 6 & 3 & 6 & 18 & 546 \\
\cline { 2 - 7 }$(62,309$ elements) & Secant scheme & 6 & 8 & 2 & 22 & 299 \\
\hline
\end{tabular}

Table 3: CPU time and RAM memory requirements in the skew-notched cylindrical beam under torsion. The proposed method is compared with the full mixed and full irreducible formulations. Likewise, the Newton-Raphson and Secant schemes are compared per first iteration factorization time, number of iterations and step average time.

\section{Conclusion}

The mixed strain displacement $\boldsymbol{\varepsilon}-\boldsymbol{u}$ finite element method is applied to problems involving mode III and mixed mode failure in quasi-brittle materials. First, the formulation is presented for isotropic continuum damage and plasticity constitutive models. The compatibility between the proposed formulation and the standard irreducible one is established, as a mean to provide a speedup of the computational time. The proposed mixed finite element technology is able to describe effectively failure processes that involve complex crack surfaces.

The three point bending test of a skew notched beam is studied using Rankine's failure criterion. The mixed strain-displacement $\boldsymbol{\varepsilon}-\boldsymbol{u}$ formulation outperforms the standard irreducible one, which shows mesh biased localization and lack of accuracy. The characteristic failure surface twisting rotation is recovered and the numerically computed crack profile is satisfactorily close to the experimental one.

Then, the torsion test of a skew-notched prismatic beam with square cross section is studied. Firstly, using the isotropic damage and the associative plasticity with Rankine's failure criterion, it is possible to properly reproduce the propagation of fracture surface found experimentally. Secondly, the two constitutive laws are compared with the plots of the vertical force versus orthogonal and sliding CMOD. It is determined that, in the mode I failure, the orthotropic nature of plasticity results in a different global behavior than the isotropic damage. The latter one provides good results, with similar values to the experiments.

Using an identical test setup, a skew-notched cylindrical beam under torsion is studied as well. Here, using a Drucker-Prager constitutive model, the influence of shear strength in the experiment is highlighted. By introducing a Drucker-Prager law with friction angle of $45^{\circ}$, the global behavior found in experiments is accurately predicted. This shows that the mode I failure criterion has to be combined with a shear-pressure dependent one, in a mixed mode fracture fashion, to properly compute the cracking of the specimen.

Finally, the kinematic compatibility between the mixed and the standard finite elements is exploited to provide considerable gains in terms of computational time. Moreover, a symmetric scheme, such as the secant one, is demonstrated as a feasible and advantageous alternative to the Newton-Raphson method.

\section{Acknowledgments}

Financial support from the EC $7^{\text {th }}$ Framework Programme under the MuMoLaDe project - Multiscale Modelling of Landslides and Debris Flows - within the framework of Marie Curie ITN (Initial Training Networks) and the Spanish Ministry of Economy and Competitivity under the EACY project - Enhanced accuracy computational and experimental framework for strain localization and failure mechanisms- within the "Excellency" Program for Knowledge Generation is gratefully acknowledged. 


\section{References}

[1] L. P. Pook. The effect of crack angle on fracture toughness. Engineering Fracture Mechanics, 3 (3):205 - 218, 1971.

[2] L. P. Pook. The fatigue crack direction and threshold behaviour of mild steel under mixed mode I and III loading. International Journal of Fatigue, 7(1):21-30, 1985.

[3] L. P. Pook. On fatigue crack paths. International Journal of Fatigue, 17(1):5-13, 1995.

[4] J. R. Yates and R. A. Mohammed. Crack propagation under mixed mode (I+ III) loading. Fatigue E Fracture of Engineering Materials \& Structures, 19(10):1285-1290, 1996.

[5] M. L. Cooke and D. D. Pollard. Fracture propagation paths under mixed mode loading within rectangular blocks of polymethyl methacrylate. Journal of Geophysical Research: Solid Earth, 101(B2):3387-3400, 1996.

[6] V. Lazarus and J.-B. Leblond. Crack paths under mixed mode (I+ III) or (I+ II+ III) loadings. Comptes Rendus de l'Academie des Sciences Series IIB Mechanics Physics Chemistry Astronomy, 326(3):171-177, 1998.

[7] V. Lazarus, J.-B. Leblond, and S.-E. Mouchrif. Crack front rotation and segmentation in mixed mode I+III or I+II+III. Part I: Calculation of stress intensity factors. Journal of the Mechanics and Physics of Solids, 49(7):1399-1420, 2001.

[8] V. Lazarus, J.-B. Leblond, and S.-E. Mouchrif. Crack front rotation and segmentation in mixed mode I+III or I+II+III. Part II: Comparison with experiments. Journal of the Mechanics and Physics of Solids, 49(7):1421-1443, 2001.

[9] F.-G. Buchholz, A. Chergui, and H. A. Richard. Fracture analyses and experimental results of crack growth under general mixed mode loading conditions. Engineering Fracture Mechanics, 71 (4):455-468, 2004.

[10] V. Lazarus, F.-G. Buchholz, M. Fulland, and J. Wiebesiek. Comparison of predictions by mode II or mode III criteria on crack front twisting in three or four point bending experiments. International Journal of Fracture, 153(2):141-151, 2008.

[11] A. Hillerborg, M. Modéer, and P.-E. Petersson. Analysis of crack formation and crack growth in concrete by means of fracture mechanics and finite elements. Cement and Concrete Research, 6 (6):773-781, 1976.

[12] Z. P. Bažant and B. H. Oh. Crack band theory for fracture of concrete. Matériaux et Construction, 16(3):155-177, 1983.

[13] G. Pijaudier-Cabot and Z. P. Bazant. Nonlocal damage theory. Journal of engineering mechanics, 113(10):1512-1533, 1987.

[14] M. Jirasek. Nonlocal models for damage and fracture: comparison of approaches. International Journal of Solids and Structures, 35(31):4133-4145, 1998.

[15] R. H. J. Peerlings, R. De Borst, W. A. M. Brekelmans, and M. G. D. Geers. Gradient-enhanced damage modelling of concrete fracture. Mechanics of Cohesive-frictional Materials, 3(4):323-342, 1998.

[16] A. Simone, G. N. Wells, and L. J. Sluys. From continuous to discontinuous failure in a gradientenhanced continuum damage model. Computer Methods in Applied Mechanics and Engineering, 192(41):4581-4607, 2003. 
[17] P. Steinmann and K. Willam. Localization within the framework of micropolar elasto-plasticity. In Advances in continuum mechanics, pages 296-313. Springer, 1991.

[18] H. B. Mühlhaus and I. Vardoulakis. The thickness of shear bands in granular materials. Geotechnique, 37(3):271-283, 1987.

[19] C. Miehe, F. Welschinger, and M. Hofacker. Thermodynamically consistent phase-field models of fracture: Variational principles and multi-field fe implementations. International Journal for Numerical Methods in Engineering, 83(10):1273-1311, 2010.

[20] J. Vignollet, S. May, R. De Borst, and C. V. Verhoosel. Phase-field models for brittle and cohesive fracture. Meccanica, 49(11):2587-2601, 2014.

[21] M. Cervera, E. Hinton, and O. Hassan. Nonlinear analysis of reinforced concrete plate and shell structures using 20-noded isoparametric brick elements. Computers $\& 3$ structures, 25(6):845-869, 1987.

[22] D. V. Swenson and A. R. Ingraffea. Modeling mixed-mode dynamic crack propagation nsing finite elements: theory and applications. Computational Mechanics, 3(6):381-397, 1988.

[23] T. Belytschko and T. Black. Elastic crack growth in finite elements with minimal remeshing. International journal for numerical methods in engineering, 45(5):601-620, 1999.

[24] J. C. Simo and M. S. Rifai. A class of mixed assumed strain methods and the method of incompatible modes. International journal for numerical methods in engineering, 29(8):1595$1638,1990$.

[25] J. C. Simo and F. Armero. Geometrically non-linear enhanced strain mixed methods and the method of incompatible modes. International Journal for Numerical Methods in Engineering, 33 (7):1413-1449, 1992.

[26] N. Sukumar, D. L. Chopp, N. Moës, and T. Belytschko. Modeling holes and inclusions by level sets in the extended finite-element method. Computer methods in applied mechanics and engineering, 190(46):6183-6200, 2001.

[27] N. Moës and T. Belytschko. Extended finite element method for cohesive crack growth. Engineering fracture mechanics, 69(7):813-833, 2002.

[28] J. Oliver, M. Cervera, and O. Manzoli. Strong discontinuities and continuum plasticity models: the strong discontinuity approach. International journal of plasticity, 15(3):319-351, 1999.

[29] F. Armero and C. Linder. New finite elements with embedded strong discontinuities in the finite deformation range. Computer Methods in Applied Mechanics and Engineering, 197(33): 3138-3170, 2008.

[30] M. Cervera, M. Chiumenti, Q. Valverde, and C. Agelet de Saracibar. Mixed linear/linear simplicial elements for incompressible elasticity and plasticity. Computer Methods in Applied Mechanics and Engineering, 192(49-50):5249-5263, 122003.

[31] M. Cervera, M. Chiumenti, and C. Agelet de Saracibar. Shear band localization via local J2 continuum damage mechanics. Computer Methods in Applied Mechanics and Engineering, 193 (9-11):849-880, March 2004.

[32] M. Cervera, M. Chiumenti, and R. Codina. Mixed stabilized finite element methods in nonlinear solid mechanics. Part I: Formulation. Computer Methods in Applied Mechanics and Engineering, 199(37-40):2559-2570, August 2010. 
[33] M. Cervera, M. Chiumenti, and R. Codina. Mixed stabilized finite element methods in nonlinear solid mechanics. Part II: Strain localization. Computer Methods in Applied Mechanics and Engineering, 199(37-40):2571-2589, August 2010.

[34] M. Cervera, M. Chiumenti, and R. Codina. Mesh objective modeling of cracks using continuous linear strain and displacement interpolations. International Journal for Numerical Methods in Engineering, 87(10):962-987, 2011.

[35] L. Benedetti, M. Cervera, and M. Chiumenti. High-fidelity prediction of crack formation in 2D and 3D pullout tests. Computers \&5 Structures, 172:93 - 109, 2016.

[36] M. Cervera, M. Chiumenti, L. Benedetti, and R. Codina. Mixed stabilized finite element methods in nonlinear solid mechanics. Part III: Compressible and Incompressible Plasticity. Computer Methods in Applied Mechanics and Engineering, 285:752-775, 2015.

[37] L. Benedetti, M. Cervera, and M. Chiumenti. Stress-accurate Mixed FEM for soil failure under shallow foundations involving strain localization in plasticity. Computers and Geotechnics, 64: $32-47,2015$.

[38] R. Faria, J. Oliver, and M. Cervera. A strain-based plastic viscous-damage model for massive concrete structures. International Journal of Solids and Structures, 35(14):1533-1558, 1998.

[39] I. Babuška. Error-bounds for finite element method. Numerische Mathematik, 16:322-333, 1971.

[40] F. Brezzi. On the existence, uniqueness and approximation of saddle-point problems arising from lagrangian multipliers. ESAIM: Mathematical Modelling and Numerical Analysis - Modlisation Mathmatique et Analyse Numrique, 8(R2):129-151, 1974.

[41] T. J. R. Hughes, L. P. Franca, and M. Balestra. A new finite element formulation for computational fluid dynamics: V. Circumventing the Babuška-Brezzi condition: A stable Petrov-Galerkin formulation of the Stokes problem accommodating equal-order interpolations. Computer Methods in Applied Mechanics and Engineering, 59(1):85-99, 1986.

[42] D. Boffi, F. Brezzi, and M. Fortin. Mixed Finite Element Methods and Applications. Springer, 2013 edition, 72013.

[43] T. J. R. Hughes, G. R. Feijóo, L. Mazzei, and J. B. Quincy. The variational multiscale method - a paradigm for computational mechanics. Computer Methods in Applied Mechanics and Engineering, 7825(98), 1998.

[44] R. Codina. Stabilization of incompressibility and convection through orthogonal sub-scales in finite element methods. Computer Methods in Applied Mechanics and Engineering, 190:1579$1599,2000$.

[45] M. Cervera, L. Pelà, R. Clemente, and P. Roca. A crack-tracking technique for localized damage in quasi-brittle materials. Engineering Fracture Mechanics, 77(13):2431-2450, 2010.

[46] M. Cervera and M. Chiumenti. Mesh objective tensile cracking via a local continuum damage model and a crack tracking technique. Computer Methods in Applied Mechanics and Engineering, 196(1):304-320, 2006.

[47] R. Citarella and F.-G. Buchholz. Comparison of crack growth simulation by DBEM and FEM for SEN-specimens undergoing torsion or bending loading. Engineering Fracture Mechanics, 75 (3):489-509, 2008.

[48] G. Ferté, P. Massin, and N. Moës. 3D crack propagation with cohesive elements in the extended finite element method. Computer Methods in Applied Mechanics and Engineering, 300:347-374, 2016. 
[49] A. D. Jefferson, B. I. G. Barr, T. Bennett, and S. C. Hee. Three dimensional finite element simulations of fracture tests using the Craft concrete model. Computers and Concrete, 1(3): 261-284, 2004.

[50] F.-G. Buchholz, V. Just, and H. A. Richard. Computational simulation and experimental findings of three-dimensional fatigue crack growth in a single-edge notched specimen under torsion loading. Fatigue $\mathcal{G}$ Fracture of Engineering Materials $\&$ Structures, 28(1-2):127-134, 2005.

[51] S. Saloustros, L. Pelà, and M. Cervera. A crack-tracking technique for localized cohesive-frictional damage. Engineering Fracture Mechanics, 150:96-114, 2015. 